\title{
Mixed shell element for seven-parameter formulation
}

\author{
Youngwon Hahn*,† and Noboru Kikuchi ${ }^{\ddagger}$ \\ Department of Mechanical Engineering, University of Michigan, 2350 Hayward, Room 3005 EECS, \\ Ann Arbor, MI 48109, U.S.A.
}

\begin{abstract}
SUMMARY
A new mixed shell element is developed for a seven-parameter formulation in this paper. The mixed shell element is constructed by assuming stress field and displacement field together. Assumed stress field and assumed displacement field can be combined by stress-strain relationship with Hu-Washizu functional. The developed mixed shell element can provide more flexible stiffness than other commercial softwares. Additionally, seven-parameter shell formulation is used instead of Reissner/Mindlin formulation, since it can provide the thickness change. Even though some commercial engineering software are not proper for very thick shell structure, the developed mixed shell element for sevenparameter formulation can be used without distinction of thick shell and thin shell. An example of shell models with different thickness is provided with solid model. Static and modal analyses are also performed for verification. Copyright (C) 2005 John Wiley \& Sons, Ltd.
\end{abstract}

KEY WORDS: mixed shell element; seven-parameter shell formulation; thickness change; orthogonal condition; Hu-Washizu functional; assumed stress

\section{INTRODUCTION}

It took many years of struggle for engineers and researchers to make an efficient shell element. Among various shell elements, low-order shell element is more popular than high-order element due to its convenience to construct model and low cost of computation. It can be applied in many industrial fields, such as automotive, aerospace, and even MEMS.

Many efforts have been made to make an efficient shell element in the past years. Even though it is impossible to present all the related papers, it is meaningful to list some important

\footnotetext{
${ }^{*}$ Correspondence to: Y. Hahn, Department of Mechanical Engineering, University of Michigan, 2350 Hayward, Room 3005 EECS, Ann Arbor, MI 48109, U.S.A.

†E-mail: yhahn@umich.edu

‡E-mail: kikuchi@umich.edu

Contract/grant sponsor: ARC (Automotive Research Center)
}

Copyright (c) 2005 John Wiley \& Sons, Ltd.

Received 15 October 2004

Revised 21 January 2005

Accepted 2 March 2005 
contributors for it: Ahmad introduced the degenerated shell element from solid element [1]. Since this element can be derived directly from the fundamental three-dimensional continuum mechanics, it has been very popular over the past years. However, the computing cost is expensive and it has some limitations to the non-linear analysis; Argyris [2,3] developed a new triangle element that used the three axes-which is called the natural co-ordinate-which are parallel to the sides of triangle. This method does not need expensive numerical integration, since the stiffness method is derived with a simple algebraic expression. It also eliminates shear-locking effect; Hughes developed a reduced integration scheme [4] and Belytschko et al. introduced one-point integration and the stabilization scheme for element's rank deficiency [5]. LS-DYNA used Belytschko's shell element and provides fast calculation; Simo re-examined the incompatible mode approach and developed assumed enhanced strain element [6,7]. This kind of assumed strain method is widely used [8-10]; even though assumed strain method is known as an old method, assumed stress method is a much older method [11-13]. In fact, it can be said that the assumed stress method is the first finite element technique. Recently, Sekiguchi focused on this method and developed the distortion-free element [14].

In this paper, mixed shell element is considered. Instead of assuming the strain part only, an assumed stress field is also used at the same time. Two assumed fields can be combined by stress-strain relationship with $\mathrm{Hu}$-Washizu functional.

In order to enhance the drawback of conventional shell formulation, seven-parameter shell formulation $[15,16]$ is considered in this paper. Conventional shell formulation, which is Reissner/Mindlin formulation [17,18], cannot deal with the thickness change, since constant thickness is assumed. This effects the conventional shell formulation which cannot handle very thick shell structure. In order to deal with either the thick shell or thin shell, seven-parameter shell formulation $[15,16]$ is considered. Even though six-parameter shell formulation is dealt with thickness change, it has a problem of thickness locking. In order to cure the thickness locking, seven-parameter shell formulation should be used. The mixed shell element is developed for this seven-parameter formulation.

The organization of this paper is as follows. First, a seven-parameter shell formulation is introduced based on Bischoff [15]. Second, the mixed shell element is developed for sevenparameter formulation. Finally, several interesting numerical solution is compared with the analytical solution and other commercial software. Small deformation and linear elastic and isotropic materials are considered in this paper.

\section{SEVEN-PARAMETER SHELL FORMULATION}

\section{Kinematics}

Figure 1 shows the original and deformed configurations of the middle surface of a shell structure. $\mathbf{a}_{i}$ is the unit direction vector and $\theta^{i}$ is the surface co-ordinate (the symbol $i$ describes corresponding co-ordinate direction). Then, the kinematics of seven-parameter shell formulation can be defined as follows [15]:

$$
\mathbf{x}=\mathbf{r}+\theta^{3} \mathbf{a}_{3}, \quad \overline{\mathbf{x}}=\overline{\mathbf{r}}+\theta^{3} \overline{\mathbf{a}}_{3}, \quad \overline{\mathbf{r}}=\mathbf{r}+\mathbf{v}, \quad \overline{\mathbf{a}}_{3}=\mathbf{a}_{3}+\mathbf{w}
$$




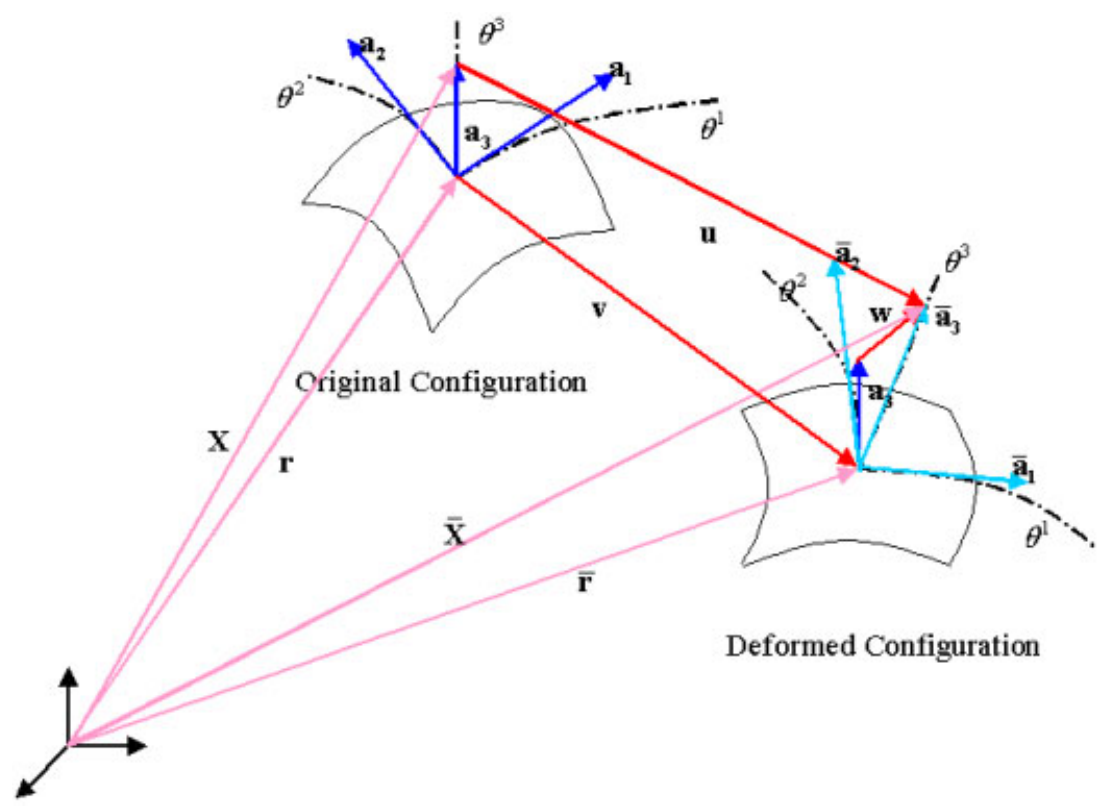

Figure 1. Kinematics of seven-parameter shell formulation.

where the bar describes the deformed configuration. Each variable is the function of the surface co-ordinate:

$$
\begin{aligned}
& \overline{\mathbf{x}}=\overline{\mathbf{x}}\left(\theta^{1}, \theta^{2}, \theta^{3}\right), \quad \mathbf{x}=\mathbf{x}\left(\theta^{1}, \theta^{2}, \theta^{3}\right) \\
& \overline{\mathbf{r}}=\overline{\mathbf{r}}\left(\theta^{1}, \theta^{2}\right), \quad \mathbf{r}=\mathbf{r}\left(\theta^{1}, \theta^{2}\right), \quad \mathbf{a}_{3}=\mathbf{a}_{3}\left(\theta^{1}, \theta^{2}\right), \quad \overline{\mathbf{a}}_{3}=\overline{\mathbf{a}}_{3}\left(\theta^{1}, \theta^{2}\right)
\end{aligned}
$$

The unit normal vector of the surface can be also defined as

$$
\mathbf{a}_{\alpha}=\frac{\partial \mathbf{r}}{\partial \theta^{\alpha}}=\mathbf{r}_{, \alpha} \quad \text { and } \quad \mathbf{a}_{3}=\frac{h}{2} \frac{\mathbf{a}_{1} \times \mathbf{a}_{2}}{\left|\mathbf{a}_{1} \times \mathbf{a}_{2}\right|}, \quad h: \text { shell thickness }
$$

Using the above relationships, the displacement vector can be obtained as follows:

$$
\mathbf{u}=\overline{\mathbf{x}}-\mathbf{x}=\mathbf{r}+\mathbf{v}+\theta^{3}\left(\mathbf{a}_{3}+\mathbf{w}\right)-\mathbf{r}-\theta^{3} \mathbf{a}_{3}=\mathbf{v}+\theta^{3} \mathbf{w}
$$

If we assume that $\mathbf{w}$ is just a rotation vector, this kinematic equation represents Reissner/Mindlin formulation, which is called five-parameter shell formulation, (Equation (5)). However, considering $\mathbf{w}$ as a translational vector that represents the thickness change and rotation, the kinematic equation for seven-parameter shell formulation can be obtained (Equation (6)).

$$
\left\{\begin{array}{l}
u_{x} \\
u_{y} \\
u_{z}
\end{array}\right\}=\left\{\begin{array}{c}
v_{x} \\
v_{y} \\
v_{z}
\end{array}\right\}+\theta^{3}\left\{\begin{array}{l}
w_{x} \\
w_{y}
\end{array}\right\}
$$




$$
\left\{\begin{array}{l}
u_{x} \\
u_{y} \\
u_{z}
\end{array}\right\}=\left\{\begin{array}{c}
v_{x} \\
v_{y} \\
v_{z}
\end{array}\right\}+\theta^{3}\left\{\begin{array}{c}
w_{x} \\
w_{y} \\
w_{z}
\end{array}\right\}
$$

where $w_{z}$ in Equation (6) is the thickness change and the variables of $w_{x}$ and $w_{y}$ are just translation vectors which describe rotational behaviour.

The strain tensor can be obtained using Green-Lagrange strain tensor that is dependent on displacement [15].

$$
\begin{aligned}
& \mathbf{E}_{i j}=\mathbf{E}_{i j}^{u} \quad(i, j=1,2,3) \\
& \mathbf{E}_{i j}^{u}=\frac{1}{2}\left(\mathbf{g}_{i} \bullet \mathbf{u}_{, j}+\mathbf{g}_{j} \bullet \mathbf{u}_{, i}+\mathbf{u}_{, j} \bullet \mathbf{u}_{, i}\right)
\end{aligned}
$$

where $\mathbf{g}_{\alpha}=\mathbf{x}_{, \alpha}=\mathbf{r}_{, \alpha}+\theta^{3} \mathbf{a}_{3, \alpha}$ and $\mathbf{g}_{3}=\mathbf{x}_{, 3}=\mathbf{a}_{3}$. In seven-parameter shell formulation, the strain tensor is defined differently by adding additional enhanced assumed strain field $\tilde{\mathbf{E}}_{33}$ to prevent the thickness locking. This additional strain is known as a seventh parameter $[15,16,19]$.

$$
\begin{aligned}
& \mathbf{E}_{i j}=\mathbf{E}_{i j}^{u}+\tilde{\mathbf{E}}_{i j} \quad(i, j=1,2,3) \\
& \mathbf{E}_{i j}^{u}=\frac{1}{2}\left(\mathbf{g}_{i} \cdot \mathbf{u}_{, j}+\mathbf{g}_{j} \cdot \mathbf{u}_{, i}+\mathbf{u}_{, j} \cdot \mathbf{u}_{, i}\right) \\
& \tilde{\mathbf{E}}_{k l}=0 \quad(k, l) \neq(3,3)
\end{aligned}
$$

Therefore, we can symbolize the above equation as follows:

$$
\mathbf{E}_{i j}^{u}=\alpha_{i j}+\frac{h}{2} \theta^{3} \beta_{i j}+\frac{h^{2}}{4}\left(\theta^{3}\right)^{2} \gamma_{i j}
$$

With the small displacement assumption, the symbol of $\mathbf{E}_{i j}$ can be changed to $\varepsilon_{i j}$ and the higher order terms can be ignored [15].

$$
\varepsilon_{i j}=\alpha_{i j}+\theta^{3} \beta_{i j}
$$

The detailed strain components can be found in Reference [15].

\section{Stiffness matrix}

In order to construct the complete three-dimensional shell formulation, Hu-Washizu functional, which can make three-field formulation, is used instead of strain potential energy that is only dependent on displacement field. The Hu-Washizu functional for small deformation and linear elastic material can be described as follows [20]:

$$
\Pi_{\mathrm{HW}}=\int \frac{1}{2} \boldsymbol{\varepsilon}^{\mathrm{T}} \mathbf{D} \boldsymbol{\varepsilon}-\boldsymbol{\varepsilon}^{\mathrm{T}} \mathbf{D} \boldsymbol{\varepsilon}_{0}+\boldsymbol{\varepsilon}^{\mathrm{T}} \boldsymbol{\sigma}_{0}+\boldsymbol{\sigma}^{\mathrm{T}}(\mathbf{L} \mathbf{u}-\boldsymbol{\varepsilon}) \mathrm{d} \boldsymbol{\Omega}-\Pi_{\mathrm{ext}}
$$

where $\mathbf{L}$ is strain displacement operator, $\mathbf{D}$ is elastic modulus coefficient, $\boldsymbol{\varepsilon}$ is strain, $\boldsymbol{\varepsilon}_{0}$ is initial strain, $\sigma_{0}$ is initial stress, and $\Pi_{\text {ext }}$ is external work. If the strain field is assumed as Equation (12) and no initial strain and stress, Equation (13) can be obtained from Equation (11). 
Equation (14) represents the constitutive equation for linear material.

$$
\begin{aligned}
\boldsymbol{\varepsilon} & =\mathbf{L u}+\tilde{\boldsymbol{\varepsilon}} \\
\Pi_{\mathrm{HW}} & =\int \frac{1}{2} \boldsymbol{\varepsilon}^{\mathrm{T}} \mathbf{D} \boldsymbol{\varepsilon}+\boldsymbol{\sigma}^{\mathrm{T}}(\mathbf{L u}-\boldsymbol{\varepsilon}) \mathrm{d} \boldsymbol{\Omega}-\Pi_{\mathrm{ext}} \\
\boldsymbol{\sigma} & =\mathbf{D} \boldsymbol{\varepsilon}
\end{aligned}
$$

Then, the following weak form can be obtained:

$$
\delta \Pi_{\mathrm{HW}}=\int \delta \boldsymbol{\varepsilon}^{\mathrm{T}} \mathbf{D} \boldsymbol{\varepsilon}-\delta \boldsymbol{\sigma}^{\mathrm{T}} \tilde{\boldsymbol{\varepsilon}}+\boldsymbol{\sigma}^{\mathrm{T}}(\mathbf{L} \delta \mathbf{u})-\boldsymbol{\sigma}^{\mathrm{T}} \delta \boldsymbol{\varepsilon} \mathrm{d} \boldsymbol{\Omega}-\delta \Pi_{\mathrm{ext}}=0
$$

It can be derived that the additional incompatible strain field $\tilde{\boldsymbol{\varepsilon}}$ is to satisfy the orthogonality condition from the maximization of saddle point problem $[14,16]$.

$$
\int \delta \boldsymbol{\sigma}^{\mathrm{T}} \tilde{\boldsymbol{\varepsilon}} \mathrm{d} \Omega=0
$$

The following equation can be described from Equation (15) with Equations (12)-(14):

$$
\delta \Pi_{\mathrm{HW}}=\left[\int \delta \boldsymbol{\varepsilon}^{\mathrm{T}}(\mathbf{D} \boldsymbol{\varepsilon}-\boldsymbol{\sigma})-\delta \boldsymbol{\sigma}^{\mathrm{T}} \tilde{\boldsymbol{\varepsilon}}+(\mathbf{L} \delta \mathbf{u})^{\mathrm{T}} \boldsymbol{\sigma} \mathrm{d} \Omega\right]-\left[\int \delta \mathbf{u}^{\mathrm{T}} \mathbf{b} \mathrm{d} \boldsymbol{\Omega}+\int \delta \mathbf{u}^{\mathrm{T}} \mathbf{t} \mathrm{d} A\right]=0
$$

where $\mathbf{b}$ is body force and $\mathbf{t}$ is traction force. The external force is described as body force and traction force in Equation (17). Using Equations (14) and (16), Equation (17) turns to the general weak form for linear static analysis:

$$
\left[\int(\mathbf{L} \delta \mathbf{u})^{\mathrm{T}} \boldsymbol{\sigma} \mathrm{d} \Omega\right]=\left[\int \delta \mathbf{u}^{\mathrm{T}} \mathbf{b} \mathrm{d} \Omega+\int \delta \mathbf{u}^{\mathrm{T}} \mathbf{t} \mathrm{d} A\right]
$$

Instead of using Equation (18), the generalized Equation (19) is also useful [20].

$$
\begin{aligned}
{\left[\int \delta \boldsymbol{\varepsilon}^{\mathrm{T}}(\mathbf{D} \boldsymbol{\varepsilon}-\boldsymbol{\sigma})-\delta \boldsymbol{\sigma}^{\mathrm{T}} \tilde{\boldsymbol{\varepsilon}}+(\mathbf{L} \delta \mathbf{u})^{\mathrm{T}} \boldsymbol{\sigma} \mathrm{d} \Omega\right]-\left[\int \delta \mathbf{u}^{\mathrm{T}} \mathbf{b} \mathrm{d} \boldsymbol{\Omega}+\int \delta \mathbf{u}^{\mathrm{T}} \mathbf{t} \mathrm{d} A\right] } & =0 \\
{\left[\int \delta \boldsymbol{\varepsilon}^{\mathrm{T}}(\mathbf{D} \boldsymbol{\varepsilon}-\boldsymbol{\sigma}) \mathrm{d} \Omega\right] } & =0 \\
{\left[\int \delta \boldsymbol{\sigma}^{\mathrm{T}} \tilde{\boldsymbol{\varepsilon}} \mathrm{d} \Omega\right] } & =0
\end{aligned}
$$

Based on the displacement method, the finite element formulation for a seven-parameter shell model can be derived. Using Equation (10), the following form can be derived:

$$
\begin{aligned}
{\left[\int \delta \boldsymbol{\varepsilon}^{\mathrm{T}} \boldsymbol{\sigma} \mathrm{d} \Omega\right] } & =\left[\int \delta\left(\boldsymbol{\alpha}+\theta^{3} \boldsymbol{\beta}\right)^{\mathrm{T}} \boldsymbol{\sigma} \mathrm{d} \Omega\right]=\left[\int \delta \boldsymbol{\alpha}^{\mathrm{T}} \boldsymbol{\sigma}+\theta^{3} \delta \boldsymbol{\beta}^{\mathrm{T}} \boldsymbol{\sigma} \mathrm{d} \Omega\right] \\
& =\left[\int \delta \boldsymbol{\alpha}^{\mathrm{T}} \int \boldsymbol{\sigma} \mathrm{d} \theta^{3} \mathrm{~d} A+\int \delta \boldsymbol{\beta}^{\mathrm{T}} \int \theta^{3} \boldsymbol{\sigma} \mathrm{d} \theta^{3} \mathrm{~d} A\right]=\left[\int \delta \boldsymbol{\alpha}^{\mathrm{T}} \mathbf{n}+\delta \boldsymbol{\beta}^{\mathrm{T}} \mathbf{m} \mathrm{d} A\right]
\end{aligned}
$$


where $\mathbf{n}$ and $\mathbf{m}$ are stress resultants. Stress resultant is the integration of stress in thickness direction as shown in Equation (20). Therefore, full expression of weak form is as follows:

$$
\begin{aligned}
& {\left[\int \left\{\delta \alpha_{11} n^{11}+\delta \alpha_{22} n^{22}+\delta \alpha_{33} n^{33}+2\left(\delta \alpha_{12} n^{12}+\delta \alpha_{13} n^{13}+\delta \alpha_{23} n^{23}\right)\right.\right.} \\
& \left.\left.+\delta \beta_{11} m^{11}+\delta \beta_{22} m^{22}+\delta \beta_{33} m^{33}+2\left(\delta \beta_{12} m^{12}+\delta \beta_{13} m^{13}+\delta \beta_{23} m^{23}\right)\right\} \mathrm{d} A\right] \\
& =\left[\int \delta \mathbf{u}^{\mathrm{T}} \mathbf{b} \mathrm{d} \Omega+\int \delta \mathbf{u}^{\mathrm{T}} \mathbf{t} \mathrm{d} A\right]
\end{aligned}
$$

where $\beta_{33}$ is an additional enhanced assumed strain which is orthogonal to the stress.

Then, the discretization is possible for finite element method with additional incompatible strain field discretized as follows [16]:

$$
\beta_{33}=\alpha_{1}+\alpha_{2} \xi+\alpha_{3} \eta+\alpha_{4} \xi \eta=\left\{\begin{array}{llllll}
1 & \xi & \eta & \xi \eta
\end{array}\right\}\left\{\alpha_{1} \quad \alpha_{2} \quad \alpha_{3} \quad \alpha_{4}\right\}^{\mathrm{T}}=\mathbf{H} \boldsymbol{\alpha}
$$

where $\boldsymbol{\alpha}$ is dummy variable to describe incompatible strain field. From the above equations, the final strain-displacement relation matrix can be obtained as follows:

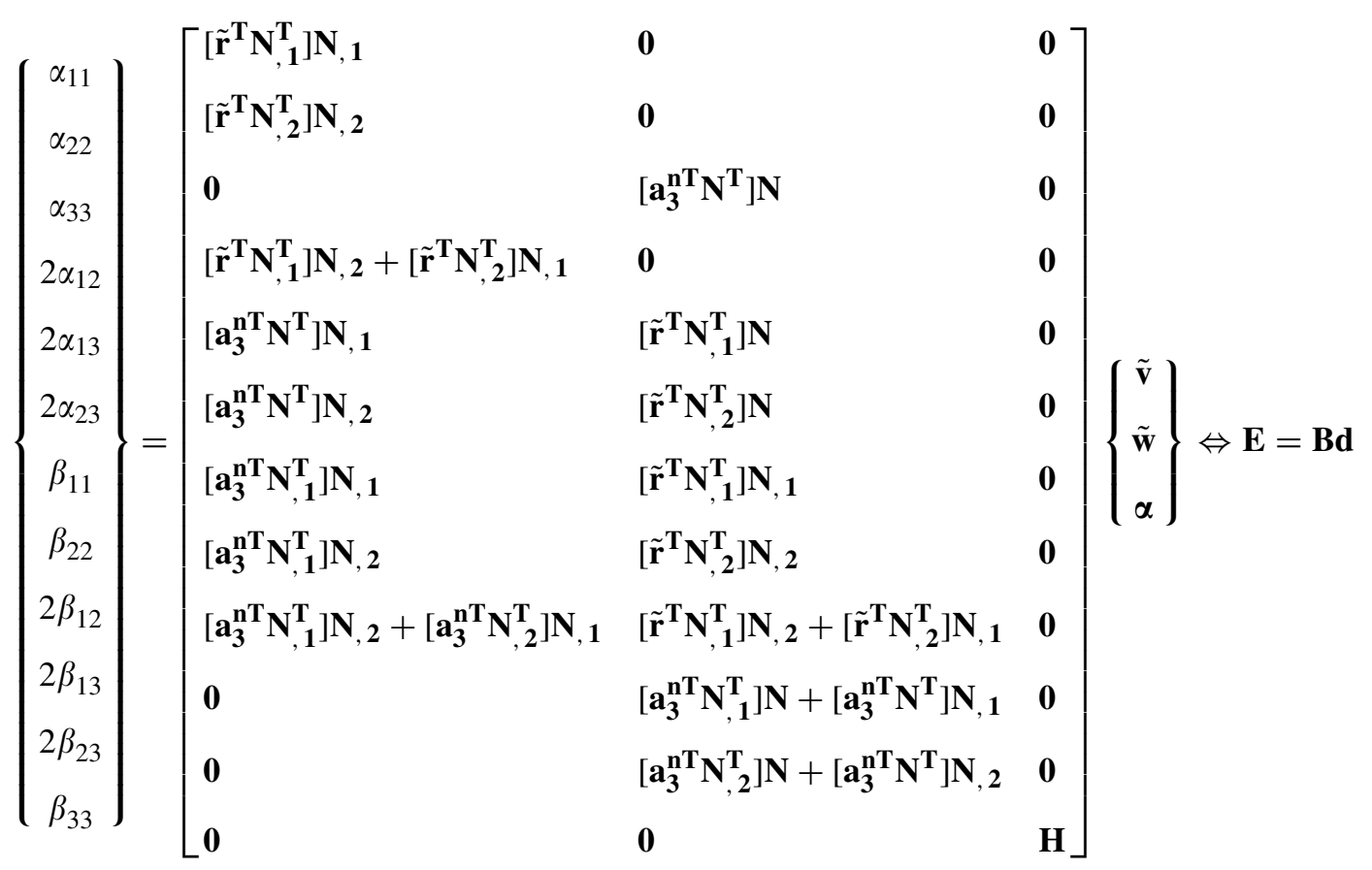

where $\mathbf{r}=\mathbf{N} \tilde{\mathbf{r}}, \mathbf{a}_{3}=\mathbf{N a}_{\mathbf{3}}, \mathbf{v}=\mathbf{N} \tilde{\mathbf{v}}$, and $\mathbf{w}=\mathbf{N} \tilde{\mathbf{w}}$. 
And stress resultant can be described in terms of strain as follows:

$$
\left\{\begin{array}{l}
n_{11} \\
n_{22} \\
n_{33} \\
n_{12} \\
n_{13} \\
n_{23} \\
m_{11} \\
m_{22} \\
m_{12} \\
m_{13} \\
m_{23} \\
m_{33}
\end{array}\right\}=\left[\begin{array}{cccccccccccc}
\bar{E} h & \lambda h & \lambda h & 0 & 0 & 0 & 0 & 0 & 0 & 0 & 0 & 0 \\
\lambda h & \bar{E} h & \lambda h & 0 & 0 & 0 & 0 & 0 & 0 & 0 & 0 & 0 \\
\lambda h & \lambda h & \bar{E} h & 0 & 0 & 0 & 0 & 0 & 0 & 0 & 0 & 0 \\
0 & 0 & 0 & G h & 0 & 0 & 0 & 0 & 0 & 0 & 0 & 0 \\
0 & 0 & 0 & 0 & G h_{q} & 0 & 0 & 0 & 0 & 0 & 0 & 0 \\
0 & 0 & 0 & 0 & 0 & G h_{q} & 0 & 0 & 0 & 0 & 0 & 0 \\
0 & 0 & 0 & 0 & 0 & 0 & \bar{E} \bar{h} & \lambda \bar{h} & 0 & 0 & 0 & \lambda \bar{h} \\
0 & 0 & 0 & 0 & 0 & 0 & \lambda \bar{h} & \bar{E} \bar{h} & 0 & 0 & 0 & \lambda \bar{h} \\
0 & 0 & 0 & 0 & 0 & 0 & 0 & 0 & G \bar{h} & 0 & 0 & 0 \\
0 & 0 & 0 & 0 & 0 & 0 & 0 & 0 & 0 & G \bar{h}_{q} & 0 & 0 \\
0 & 0 & 0 & 0 & 0 & 0 & 0 & 0 & 0 & 0 & G \bar{h}_{q} & 0 \\
0 & 0 & 0 & 0 & 0 & 0 & \lambda \bar{h} & \lambda \bar{h} & 0 & 0 & 0 & \bar{E} \bar{h}
\end{array}\right]\left\{\begin{array}{c}
\alpha_{11} \\
\alpha_{22} \\
\alpha_{33} \\
2 \alpha_{12} \\
2 \alpha_{13} \\
2 \alpha_{23} \\
\beta_{11} \\
\beta_{22} \\
2 \beta_{12} \\
2 \beta_{13} \\
2 \beta_{23} \\
\beta_{33}
\end{array}\right\}=\mathbf{D E}
$$

where

$$
\begin{aligned}
& \bar{E}=\frac{E(1-v)}{(1+v)(1-2 v)}, \quad \lambda=\frac{E v}{(1+v)(1-2 v)}, \quad G=\frac{E}{2(1+v)}, \quad h_{q}=\alpha h, \\
& \bar{h}=\frac{h^{3}}{12}, \quad \bar{h}_{q}=\beta \bar{h}
\end{aligned}
$$

$\alpha, \beta$ is shear correction factor.

In Equation (23), a remedy is needed in order to prevent the ill-conditioned stiffness matrix. If Equation (24) is concerned, it is easy to recognize that the term of $\bar{h}\left(=h^{3} / 12\right)$ will cause ill-conditioned problem. Wall et al. [21] proposed scaled director conditioning (SDC) method to prevent ill-conditioned matrix. In this paper, $(2 / h)$ is multiplied to the second column of $\mathbf{B}$ and $(h / 2)$ is multiplied to $\mathbf{w}$ in Equation (23) instead of using SDC method. The concept is identical, but this is simpler and easier to implement. The normal vector $\mathbf{a}_{3}$ should be unit vector in this case. Actually, this kind of method is already used in other paper [22] but it is not presented as a tool in order to cure the ill-conditioning problem. Figure 2 shows the comparison of eigenvalues as the thickness becomes smaller. The formulation with this numerical treatment can provide reasonable results. Otherwise, non-physical eigenvalue distribution is shown.

Finally, ordinary stiffness matrix can be constructed using the above Equations (23) and (24) and the additional incompatible strain term can be removed by static condensation. 

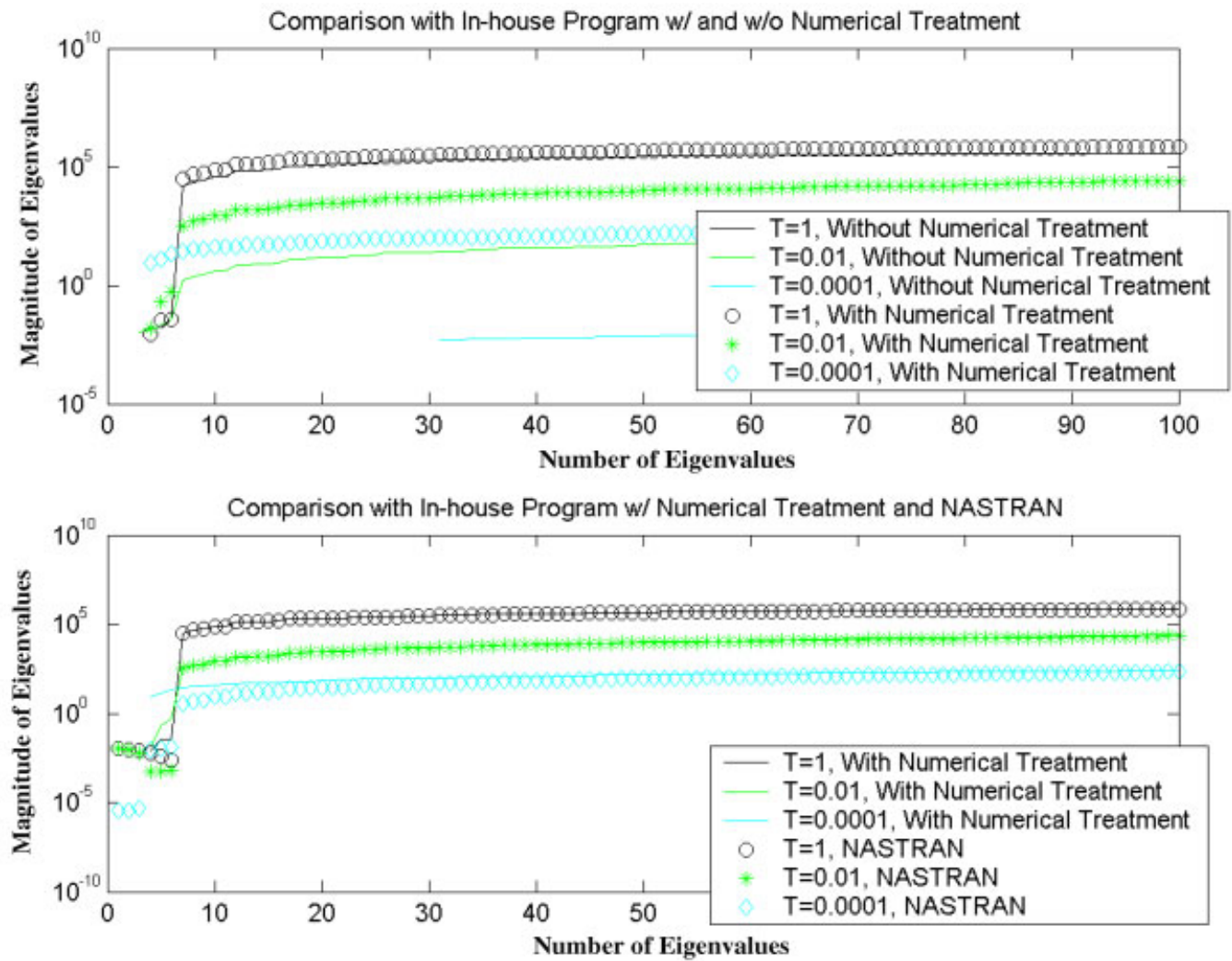

Figure 2. Comparison of eigenvalues with and without numerical treatment as thickness decreases.

\section{Mass matrix}

It can be derived from kinetic energy which is a part of external work. With the previous expressions, the mass matrix can be obtained as follows:

$$
\begin{aligned}
& \delta \mathbf{K} . \mathbf{E} .=\int\left(\delta \dot{\mathbf{u}}^{\mathrm{T}} \rho \dot{\mathbf{u}}\right) \mathrm{d} \Omega=\delta \dot{\mathbf{d}}^{\mathrm{T}} \int\left(\mathbf{N}^{\mathrm{T}} \mathbf{D}_{\rho} \mathbf{N}\right) \mathrm{d} A \dot{\mathbf{d}} \\
& =\delta\left\{\begin{array}{c}
\dot{\tilde{\mathbf{v}}} \\
\dot{\tilde{\mathbf{w}}}
\end{array}\right\}^{\mathrm{T}} \int \mathbf{N}^{\mathrm{T}}\left[\begin{array}{cccccc}
\rho h & 0 & 0 & 0 & 0 & 0 \\
0 & \rho h & 0 & 0 & 0 & 0 \\
0 & 0 & \rho h & 0 & 0 & 0 \\
0 & 0 & 0 & \rho h^{3} / 12 & 0 & 0 \\
0 & 0 & 0 & 0 & \rho h^{3} / 12 & 0 \\
0 & 0 & 0 & 0 & 0 & \rho h^{3} / 12
\end{array}\right] \mathbf{N} \mathrm{d} A\left\{\begin{array}{c}
\dot{\tilde{\mathbf{v}}} \\
\dot{\tilde{\mathbf{w}}}
\end{array}\right\}
\end{aligned}
$$


For mass matrix in Equation (25), row-sum technique is generally used for modal analysis or time integration method. Row-sum technique is to make mass matrix diagonalize-diagonal value in matrix is substituted for summation value of all members in corresponding row and every off-diagonal member is exchanged by zero. Usually, mass matrix with the same shape function used in stiffness matrix is called consistent mass matrix and mass matrix with row-sum technique is called lumped mass matrix. Generally, lumped mass matrix is used to obtain the low frequencies and consistent mass matrix is used for high frequencies.

In seven-parameter shell formulation, row-sum technique can be easily used, since all displacement vectors are described by translational vectors. However, for Reissner/Mindlin shell formulation, another technique to treat rotational vector is needed, since the independent variables are organized by translational and rotational vectors. However, the ignorance of rotational inertia term makes no difference if only the lowest eigenvalues are concerned since the rotational inertia term provides very high frequencies.

\section{MIXED SHELL ELEMENT FOR SEVEN-PARAMETER FORMULATION}

In order to make mixed shell element for seven-parameter formulation, the assumed stress field should be defined first. Based on five-parameter shell model, the assumed stress modes can be described as follows in general:

$$
\begin{aligned}
\sigma^{11} & =c_{1}+c_{2} \theta^{2}+c_{3} \theta^{3}+c_{4} \theta^{2} \theta^{3} \\
\sigma^{22} & =c_{5}+c_{6} \theta^{1}+c_{7} \theta^{3}+c_{8} \theta^{1} \theta^{3} \\
\sigma^{33} & =0 \\
\sigma^{12} & =c_{13}+c_{14} \theta^{3} \\
\sigma^{13} & =c_{15}+c_{16} \theta^{2} \\
\sigma^{23} & =c_{17}+c_{18} \theta^{1}
\end{aligned}
$$

where $c$ is constant parameter. Since five-parameter shell model assumed no thickness change, there is no stress in thickness direction. However, the thickness direction stress should be inserted in seven-parameter shell model as follows:

$$
\begin{aligned}
& \sigma^{11}=c_{1}+c_{2} \theta^{2}+c_{3} \theta^{3}+c_{4} \theta^{2} \theta^{3} \\
& \sigma^{22}=c_{5}+c_{6} \theta^{1}+c_{7} \theta^{3}+c_{8} \theta^{1} \theta^{3} \\
& \sigma^{33}=c_{9}+c_{10} \theta^{1}+c_{11} \theta^{2}+c_{12} \theta^{1} \theta^{2} \\
& \sigma^{12}=c_{13}+c_{14} \theta^{3}+c_{15} \theta^{1} \theta^{3}+c_{16} \theta^{2} \theta^{3} \\
& \sigma^{13}=c_{17}+c_{18} \theta^{2}+c_{19} \theta^{2} \theta^{1}+c_{20} \theta^{2} \theta^{3} \\
& \sigma^{23}=c_{21}+c_{22} \theta^{1}+c_{23} \theta^{1} \theta^{2}+c_{24} \theta^{1} \theta^{3}
\end{aligned}
$$

Similar assumed stress components can be found in the Sansour's work [23, 24]. 
In Equation (20), stress resultants for seven-parameter shell formulation can be derived as follows:

$$
\begin{aligned}
& n_{11}=\int_{-h / 2}^{h / 2} S_{11} \mathrm{~d} \theta^{3}=\int_{-h / 2}^{h / 2}\left(c_{1}+c_{2} \theta^{2}+c_{3} \theta^{3}+c_{4} \theta^{2} \theta^{3}\right) \mathrm{d} \theta^{3}=h\left(c_{1}+c_{2} \theta^{2}\right) \\
& n_{22}=\int_{-h / 2}^{h / 2} S_{22} \mathrm{~d} \theta^{3}=\int_{-h / 2}^{h / 2}\left(c_{5}+c_{6} \theta^{1}+c_{7} \theta^{3}+c_{8} \theta^{1} \theta^{3}\right) \mathrm{d} \theta^{3}=h\left(c_{5}+c_{6} \theta^{1}\right) \\
& n_{33}=\int_{-h / 2}^{h / 2} S_{33} \mathrm{~d} \theta^{3}=\int_{-h / 2}^{h / 2}\left(c_{9}+c_{10} \theta^{1}+c_{11} \theta^{2}+c_{12} \theta^{1} \theta^{2}\right) \mathrm{d} \theta^{3} \\
& =h\left(c_{9}+c_{10} \theta^{1}+c_{11} \theta^{2}+c_{12} \theta^{1} \theta^{2}\right) \\
& n_{12}=\int_{-h / 2}^{h / 2} S_{12} \mathrm{~d} \theta^{3}=\int_{-h / 2}^{h / 2}\left(c_{13}+c_{14} \theta^{3}+c_{15} \theta^{1} \theta^{3}+c_{16} \theta^{2} \theta^{3}\right) \mathrm{d} \theta^{3}=h\left(c_{13}\right) \\
& n_{13}=\int_{-h / 2}^{h / 2} S_{13} \mathrm{~d} \theta^{3}=\int_{-h / 2}^{h / 2}\left(c_{17}+c_{18} \theta^{2}+c_{19} \theta^{2} \theta^{1}+c_{20} \theta^{2} \theta^{3}\right) \mathrm{d} \theta^{3} \\
& =h\left(c_{17}+c_{18} \theta^{2}+c_{19} \theta^{2} \theta^{1}\right) \\
& n_{23}=\int_{-h / 2}^{h / 2} S_{23} \mathrm{~d} \theta^{3}=\int_{-h / 2}^{h / 2}\left(c_{21}+c_{22} \theta^{1}+c_{23} \theta^{1} \theta^{2}+c_{24} \theta^{1} \theta^{3}\right) \mathrm{d} \theta^{3} \\
& =h\left(c_{21}+c_{22} \theta^{1}+c_{23} \theta^{1} \theta^{2}\right) \\
& m_{11}=\int_{-h / 2}^{h / 2} \theta^{3} S_{11} \mathrm{~d} \theta^{3}=\int_{-h / 2}^{h / 2} \theta^{3}\left(c_{1}+c_{2} \theta^{2}+c_{3} \theta^{3}+c_{4} \theta^{2} \theta^{3}\right) \mathrm{d} \theta^{3}=\frac{h^{3}}{12}\left(c_{3}+c_{4} \theta^{2}\right) \\
& m_{22}=\int_{-h / 2}^{h / 2} \theta^{3} S_{22} \mathrm{~d} \theta^{3}=\int_{-h / 2}^{h / 2} \theta^{3}\left(c_{5}+c_{6} \theta^{1}+c_{7} \theta^{3}+c_{8} \theta^{1} \theta^{3}\right) \mathrm{d} \theta^{3}=\frac{h^{3}}{12}\left(c_{7}+c_{8} \theta^{1}\right) \\
& m_{33}=\int_{-h / 2}^{h / 2} \theta^{3} S_{33} \mathrm{~d} \theta^{3}=\int_{-h / 2}^{h / 2} \theta^{3}\left(c_{9}+c_{10} \theta^{1}+c_{11} \theta^{2}+c_{12} \theta^{1} \theta^{2}\right) \mathrm{d} \theta^{3}=0 \\
& m_{12}=\int_{-h / 2}^{h / 2} \theta^{3} S_{12} \mathrm{~d} \theta^{3}=\int_{-h / 2}^{h / 2} \theta^{3}\left(c_{13}+c_{14} \theta^{3}+c_{15} \theta^{1} \theta^{3}+c_{16} \theta^{2} \theta^{3}\right) \mathrm{d} \theta^{3} \\
& =\frac{h^{3}}{12}\left(c_{14}+c_{15} \theta^{1}+c_{16} \theta^{2}\right) \\
& m_{13}=\int_{-h / 2}^{h / 2} \theta^{3} S_{13} \mathrm{~d} \theta^{3}=\int_{-h / 2}^{h / 2} \theta^{3}\left(c_{17}+c_{18} \theta^{2}+c_{19} \theta^{2} \theta^{1}+c_{20} \theta^{2} \theta^{3}\right) \mathrm{d} \theta^{3}=\frac{h^{3}}{12}\left(c_{20} \theta^{2}\right) \\
& m_{23}=\int_{-h / 2}^{h / 2} \theta^{3} S_{23} \mathrm{~d} \theta^{3}=\int_{-h / 2}^{h / 2} \theta^{3}\left(c_{21}+c_{22} \theta^{1}+c_{23} \theta^{1} \theta^{2}+c_{24} \theta^{1} \theta^{3}\right) \mathrm{d} \theta^{3}=\frac{h^{3}}{12}\left(c_{24} \theta^{1}\right)
\end{aligned}
$$


where $h$ is thickness, $n$ and $m$ are stress resultants. Each stress resultant mode can be found in Reference [15].

In Equation (29), $m_{33}$ is zero. However, the enhanced assumed strain in seven-parameter formulation makes us construct the corresponding assumed stress field:

$$
m_{33}=\tilde{m}_{33}=\frac{h^{3}}{12}\left(c_{25}+c_{26} \theta^{1}+c_{27} \theta^{2}+c_{28} \theta^{1} \theta^{2}\right)
$$

Therefore, the complete assumed stress field for the seven-parameter shell model is constructed: in order to match the linear strain field in seven-parameter formulation, the linear stress field in thickness direction should be constructed as follows:

$$
\begin{aligned}
& \sigma^{11}=c_{1}+c_{2} \theta^{2}+\left(c_{3}+c_{4} \theta^{2}\right) \theta^{3} \\
& \sigma^{22}=c_{5}+c_{6} \theta^{1}+\left(c_{7}+c_{8} \theta^{1}\right) \theta^{3} \\
& \sigma^{33}=c_{9}+c_{10} \theta^{1}+c_{11} \theta^{2}+c_{12} \theta^{1} \theta^{2}+\left(c_{25}+c_{26} \theta^{1}+c_{27} \theta^{2}+c_{28} \theta^{1} \theta^{2}\right) \theta^{3} \\
& \sigma^{12}=c_{13}+\left(c_{14}+c_{15} \theta^{1}+c_{16} \theta^{2}\right) \theta^{3} \\
& \sigma^{13}=c_{17}+c_{18} \theta^{2}+c_{19} \theta^{2} \theta^{1}+c_{20} \theta^{2} \theta^{3} \\
& \sigma^{23}=c_{21}+c_{22} \theta^{1}+c_{23} \theta^{1} \theta^{2}+c_{24} \theta^{1} \theta^{3}
\end{aligned}
$$

Then, the matrix form for assumed stress can be constructed as the following matrix form:

$$
\begin{aligned}
& \mathbf{s}=\mathbf{M c} \\
& \mathbf{s}=\left\{\begin{array}{llllllllllll}
n_{11} & n_{22} & n_{33} & n_{12} & n_{13} & n_{23} & m_{11} & m_{22} & m_{12} & m_{13} & m_{23} & m_{33}
\end{array}\right\}^{\mathrm{T}} \\
& c=\left\{\begin{array}{lllllll}
c_{1} & \cdots & c_{24} & c_{25} & c_{26} & c_{27} & c_{28}
\end{array}\right\}^{\mathrm{T}}
\end{aligned}
$$

The matrix form of $\mathbf{M}$ can be found in Appendix A. Therefore, the alternate weak form can be derived from Equation (20).

$$
\begin{gathered}
\int \delta \boldsymbol{\varepsilon}^{\mathrm{T}} \mathbf{D} \boldsymbol{\varepsilon} \mathrm{d} \Omega-\left[\int \delta \mathbf{u}^{\mathrm{T}} \mathbf{b} \mathrm{d} \Omega+\int \delta \mathbf{u}^{\mathrm{T}} \mathbf{t} \mathrm{d} A\right]=0 \\
\int \delta \boldsymbol{\varepsilon}^{\mathrm{T}} \mathbf{D} \boldsymbol{\varepsilon} \mathrm{d} \Omega \Rightarrow \int \delta \boldsymbol{\varepsilon}^{\mathrm{T}} \int \boldsymbol{\sigma} \mathrm{d} \theta^{3} \mathrm{~d} A=\int \delta \boldsymbol{\alpha}^{\mathrm{T}} \int \boldsymbol{\sigma} \mathrm{d} \theta^{3}+\delta \boldsymbol{\beta}^{\mathrm{T}} \int \theta^{3} \boldsymbol{\sigma} \mathrm{d} \theta^{3} \mathrm{~d} A \\
=\int \delta \mathbf{E}^{\mathrm{T}} \mathbf{s} \mathrm{d} A=\delta \mathbf{d}^{\mathrm{T}} \int \mathbf{B}^{\mathrm{T}} \mathbf{M} \mathrm{d} A \mathbf{c} \\
{\left[\int \delta \mathbf{u}^{\mathrm{T}} \mathbf{b} \mathrm{d} \Omega+\int \delta \mathbf{u}^{\mathrm{T}} \mathbf{t} \mathrm{d} A\right] \Rightarrow \delta \mathbf{d}^{\mathrm{T}} \int \mathbf{N}^{\mathrm{T}} \mathbf{b} \mathrm{d} \Omega+\delta \mathbf{d}^{\mathrm{T}} \int \mathbf{N}^{\mathrm{T}} \mathbf{t} \mathrm{d} A=\delta \mathbf{d}^{\mathrm{T}} \mathbf{f}} \\
\therefore \int \mathbf{B}^{\mathrm{T}} \mathbf{M} \mathrm{d} A \mathbf{c}=\mathbf{f}
\end{gathered}
$$

Equation (33) is constructed by assumed stress only. In order to make mixed shell element, displacement field should be also assumed. In the previous section, the assumed displacement field is already introduced. 
The relationship between assumed stress field and displacement can be derived considering Equations (19) and (14) - it is derived directly if the complementary energy is considered.

$$
\int \delta \boldsymbol{\sigma}^{\mathrm{T}}\left(\mathbf{D}^{-1} \boldsymbol{\sigma}-\boldsymbol{\varepsilon}\right) \mathrm{d} \Omega=0 \Leftrightarrow \int \delta \boldsymbol{\sigma}^{\mathrm{T}} \mathbf{D}^{-1} \boldsymbol{\sigma} \mathrm{d} \Omega=\int \delta \boldsymbol{\sigma}^{\mathrm{T}} \boldsymbol{\varepsilon} \mathrm{d} \Omega
$$

Equation (34) can be described differently in terms of stress as follows:

$$
\begin{aligned}
\int \delta \boldsymbol{\sigma}^{\mathrm{T}} \mathbf{D}^{-1} \boldsymbol{\sigma} \mathrm{d} \Omega & =\delta \mathbf{c}^{\mathrm{T}} \int \mathbf{H}^{\mathrm{T}} \overline{\mathbf{D}}^{-1} \mathbf{H} \mathrm{d} A \mathbf{c} \\
\int \delta \boldsymbol{\sigma}^{\mathrm{T}} \boldsymbol{\varepsilon} \mathrm{d} \Omega & =\int\left(\int \delta \boldsymbol{\sigma}^{\mathrm{T}} \mathrm{d} \theta^{3} \boldsymbol{\alpha}+\int \delta \boldsymbol{\sigma}^{\mathrm{T}} \theta^{3} \mathrm{~d} \theta^{3} \boldsymbol{\beta}\right) \mathrm{d} A \\
& =\int \delta \mathbf{n}^{\mathrm{T}} \boldsymbol{\alpha}+\delta \mathbf{m}^{\mathrm{T}} \boldsymbol{\beta} \mathrm{d} A=\delta \mathbf{c}^{\mathrm{T}} \int \mathbf{M}^{\mathrm{T}} \mathbf{B} \mathrm{d} A \mathbf{d}
\end{aligned}
$$

where $\mathbf{H}$ and $\overline{\mathbf{D}}^{-1}$ are shape function matrix and inverse of elastic modulus coefficient, respectively, which are shown in Appendix A.

Therefore, Equation (34) can be described in matrix form as follows:

$$
\begin{aligned}
\int \mathbf{H}^{\mathrm{T}} \overline{\mathbf{D}}^{-1} \mathbf{H} \mathrm{d} A \mathbf{c} & =\int \mathbf{M}^{\mathrm{T}} \mathbf{B} \mathrm{d} A \mathbf{d} \Leftrightarrow \mathbf{c}=\tilde{\mathbf{M}}^{-1} \mathbf{F} \mathbf{d} \\
\tilde{\mathbf{M}} & =\int \mathbf{H}^{\mathrm{T}} \overline{\mathbf{D}}^{-1} \mathbf{H} \mathrm{d} A \\
\mathbf{F} & =\int \mathbf{M}^{\mathrm{T}} \mathbf{B} \mathrm{d} A
\end{aligned}
$$

Substituting (36) to (33),

$$
\int \mathbf{B}^{\mathrm{T}} \mathbf{M} \mathrm{d} A \mathbf{c}=\mathbf{f} \Leftrightarrow \mathbf{F}^{\mathrm{T}} \tilde{\mathbf{M}}^{-1} \mathbf{F} \mathbf{d}=\mathbf{f} \Leftrightarrow \tilde{\mathbf{K}} \mathbf{d}=\mathbf{f}
$$

Equation (37) is the final form of matrix equation for mixed shell element for seven-parameter formulation for linear elastic material.

This mixed shell element does not pass the patch test. However, the convergence is observed from several examples. In next section, various convergence examples will be provided.

\section{NUMERICAL EXAMPLES}

Commercial software, such as MSC/NASTRAN v2004, ABAQUS v6.2, and ANSYS v8.1, are used for reference. In tables, each symbol of $T x, T y$, and $T z$ stands for displacement in $x-, y$-, and $z$-direction, respectively. 


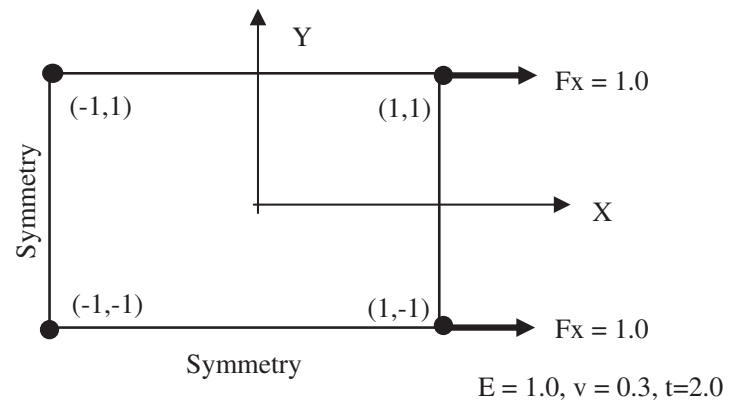

Figure 3. Finite element model for thick square plate.

Table I. The results of thick square plate.

\begin{tabular}{lcccc}
\hline & $T x$ & $T y$ & $T z$ & Thickness change \\
\hline In-house (mixed) & $\mathbf{1 . 0}$ & $\mathbf{- 0 . 3}$ & 0.0 & $\mathbf{- 0 . 1 5}$ \\
MSC/NASTRAN & $\mathbf{1 . 0}$ & $-\mathbf{0 . 3}$ & 0.0 & N/A \\
ANSYS & $\mathbf{1 . 0}$ & $\mathbf{- 0 . 3}$ & 0.0 & N/A \\
ABAQUS & $\mathbf{1 . 0}$ & $\mathbf{- 0 . 3}$ & 0.0 & N/A \\
\hline
\end{tabular}

Table II. The results of cantilever case of tension test model with constraint in the thickness direction.

\begin{tabular}{lcccc}
\hline & $T x$ & $T y$ & $T z$ & Thickness change \\
\hline In-house (mixed) & $\mathbf{0 . 9 3 6}$ & $\mathbf{- 0 . 2 2 5}$ & 0.0 & $\mathbf{- 0 . 2 2 5}$ \\
MSC/NASTRAN & $\mathbf{0 . 9 7 7}$ & $\mathbf{- 0 . 2 2 5}$ & 0.0 & N/A \\
ANSYS & $\mathbf{0 . 9 7 8}$ & $\mathbf{- 0 . 2 2 5}$ & 0.0 & N/A \\
ABAQUS & $\mathbf{0 . 9 7 7}$ & $\mathbf{- 0 . 2 2 4}$ & 0.0 & N/A \\
\hline
\end{tabular}

\section{Tension test for thick square plate}

The model is shown in Figure 3. This example is a good example to compare conventional shell formulation and seven-parameter shell formulation. Even though the shape of model in Figure 3 is two-dimensional plate, the model is a hexahedron solid since the thickness is the same as the surface dimension. Commercial software based on five-parameter shell formulation and in-house program based on seven-parameter shell formulation are tested. Considering boundary condition, the answer should be the same, since it is just tension test. Table I shows that the displacement results of forced node. The only in-house program can provide the thickness change.

If one end is fixed like a cantilever beam, the interesting result can be obtained as shown in Table II. In commercial software, the displacement in the normal direction of load is the same as that of in-house program, even though the displacement result in the loading direction is larger than that of in-house program.

Another example is to release the constraint in the thickness direction. Table III shows the results of this case. Mixed shell element provides almost the same values as other commercial 
Table III. The results of cantilever case of tension test model without constraint in the thickness direction.

\begin{tabular}{lcccc}
\hline & $T x$ & $T y$ & $T z$ & Thickness change \\
\hline In-house (mixed) & $\mathbf{0 . 9 7 7}$ & $\mathbf{- 0 . 2 2 5}$ & 0.0 & $\mathbf{- 0 . 1 2 7}$ \\
MSC/NASTRAN & $\mathbf{0 . 9 7 7}$ & $\mathbf{- 0 . 2 2 5}$ & 0.0 & N/A \\
ANSYS & $\mathbf{0 . 9 7 8}$ & $\mathbf{- 0 . 2 2 5}$ & 0.0 & N/A \\
ABAQUS & $\mathbf{0 . 9 7 7}$ & $\mathbf{- 0 . 2 2 4}$ & 0.0 & N/A \\
\hline
\end{tabular}

Table IV. The results of cantilever case of tension test model with solid element (CHEXA) of MSC/NASTRAN.

\begin{tabular}{ccccc}
\hline & $T x$ & $T y$ & $T z$ & Thickness change \\
\hline MSC/NASTRAN & $\mathbf{0 . 9 3 6}$ & $\mathbf{- 0 . 2 2 5}$ & $\mathbf{- 0 . 2 2 5}$ & N/A \\
\hline
\end{tabular}

software. Especially, the same results are provided with MSC/NASTRAN except for thickness change.

If the solid element model is tested, its result is identical to that of in-house program. Therefore, in-house program is more reasonable in physical point of view (Table IV).

\section{Shear test for plate}

This example is famous for shear test and described in Reference [25]. The model is shown in Figure 4. As shown in Table V, the result of in-house program presents similar results compared with other software. Unlike the previous example, the irregular elements are used and tested in this case. Table $\mathrm{V}$ shows the displacements of one of tip nodal points applied force. The results of in-house program are little larger than those of commercial software. However, those are acceptable in author's opinion since the error is not severe.

\section{Out-of-plane bending test for plate}

The model, which has the dimension of $6 \mathrm{~mm}$ for length, $0.2 \mathrm{~mm}$ for width, and $0.1 \mathrm{~mm}$ for thickness, is shown in Figure 5. Typical case of out-of-plane bending is examined. The results show good performance of in-house program in Table VI. Table VI shows the displacement results of tip nodes applied force. The analytical solution is 0.432 based on Reference [26]. There is only small difference between in-house and commercial software. All of programs provide good performance for out-of-plane bending case.

\section{Twisted beam}

The model is shown in Figure 6. This example is for testing a distorted shell element. Table VII shows good displacement results of in-house program which used mixed shell element compared with the analytical solution, 1.75e-3, from Reference [26] at the node applied force. ANSYS provides the largest deformation value compared with other results. 


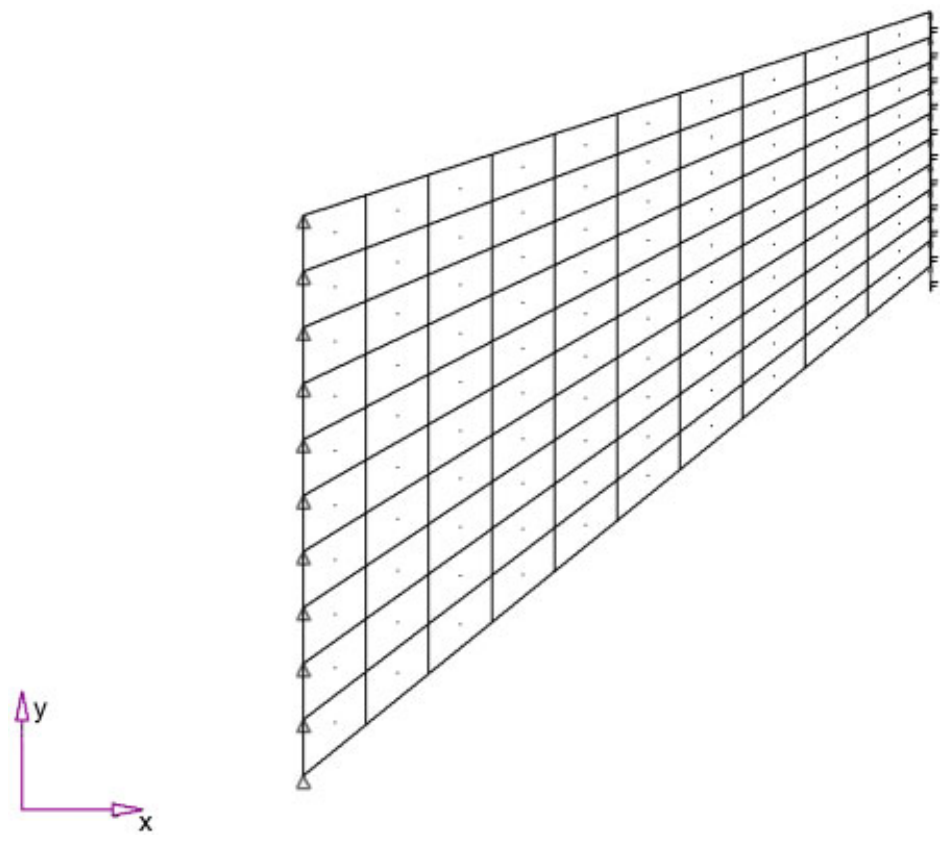

Figure 4. Finite element model for shear test $(E=2.0 \mathrm{e} 8, v=0.3, t=0.1)$.

Table V. The results of shear test.

\begin{tabular}{lccc}
\hline & $T x$ & $T y$ & $T z$ \\
\hline In-house (mixed) & $-1.272 \mathrm{e}-6$ & $\mathbf{1 . 0 9 6 7 e - 5}$ & 0.0 \\
MSC/NASTRAN & $-1.336 \mathrm{e}-6$ & $\mathbf{1 . 0 4 3 2 e - 5}$ & 0.0 \\
ANSYS & $-1.336 \mathrm{e}-6$ & $\mathbf{1 . 0 4 3 2 e - 5}$ & 0.0 \\
ABAQUS & $-1.329 \mathrm{e}-6$ & $\mathbf{1 . 0 4 7 1 e - 5}$ & 0.0 \\
\hline
\end{tabular}

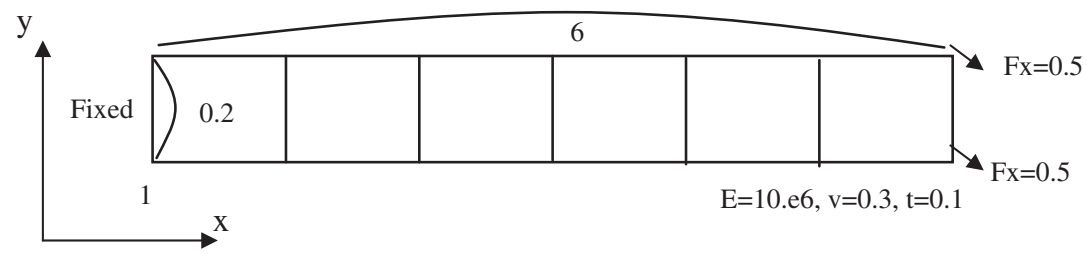

Figure 5. Finite element model for out-of-plane bending test.

\section{$18^{\circ}$ pinched hole hemisphere}

This example is famous for testing shell element. The model is shown in Figure 7. The results in Table VIII show good performance of in-house program. Table VIII shows the displacement result at the node applied force. The reference value, 0.094, is presented as an upper bound in 
Table VI. The results of out-of-plane bending test.

\begin{tabular}{lccc}
\hline & $T x$ & $T y$ & $T z$ \\
\hline In-house (mixed) & 0.0 & 0.0 & $\mathbf{0 . 4 2 4}$ \\
MSC/NASTRAN & 0.0 & 0.0 & $\mathbf{0 . 4 2 6}$ \\
ANSYS & 0.0 & 0.0 & $\mathbf{0 . 4 2 2}$ \\
ABAQUS & 0.0 & 0.0 & $\mathbf{0 . 4 2 4}$ \\
\hline
\end{tabular}
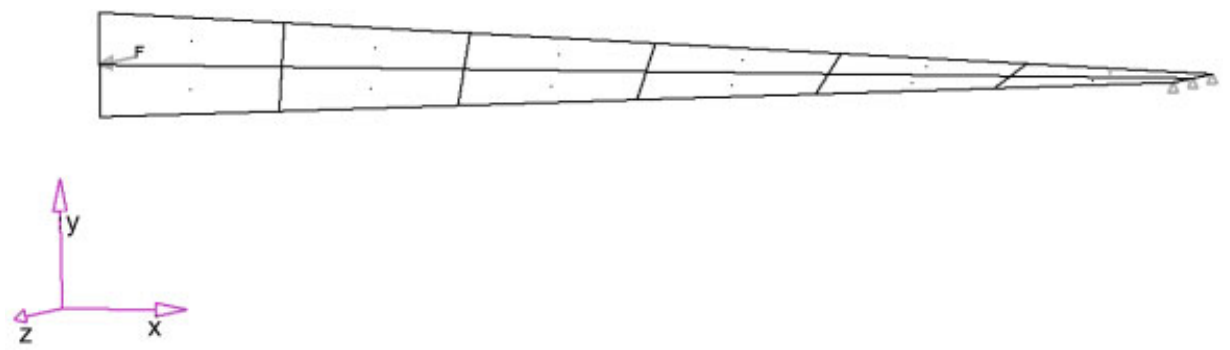

Figure 6. Finite element model for curved plate $(E=2.9 \mathrm{e} 7, v=0.22, t=0.32)$.

Table VII. The results of curved plate.

\begin{tabular}{lrcc}
\hline & \multicolumn{1}{c}{$T x$} & $T y$ & $T z$ \\
\hline In-house (mixed) & $-3.368 \mathrm{e}-10$ & $1.873 \mathrm{e}-3$ & $\mathbf{2 . 3 4 0 e - 3}$ \\
MSC/NASTRAN & $-6.564 \mathrm{e}-18$ & $1.760 \mathrm{e}-3$ & $\mathbf{2 . 2 6 3 e - 3}$ \\
ANSYS & $1.813 \mathrm{e}-11$ & $1.869 \mathrm{e}-3$ & $\mathbf{2 . 4 2 1 e - 3}$ \\
ABAQUS & $-3.285 \mathrm{e}-11$ & $1.914 \mathrm{e}-3$ & $\mathbf{2 . 2 2 7 e - 3}$ \\
\hline
\end{tabular}

Reference [26]. Considering the theoretical upper bound 0.094, in-house program has a little larger result.

\section{Open section frame}

The model is shown in Figure 8. This model is considered as a practical case that can be used in real industrial field-it is also considered in Reference [25]. This model can be used as a component of side frame of commercial vehicle. Since the analytical solution cannot be provided, the results of other softwares are compared. The displacement results at the node applied force in Table IX shows that the in-house presents good performance.

\section{Thin plate and thick plate}

Two plate models constructed by shell and solid elements are shown in Figures 9(a) and (b), respectively. Two different models that have different thicknesses are tested. Both models have $2 \mathrm{~mm}$ length and $2 \mathrm{~mm}$ width. However, one of them has $0.01 \mathrm{~mm}$ thickness and the other has $1 \mathrm{~mm}$ thickness. First, MSC/NASTRAN is used to simulate free-free modal analysis. The first 


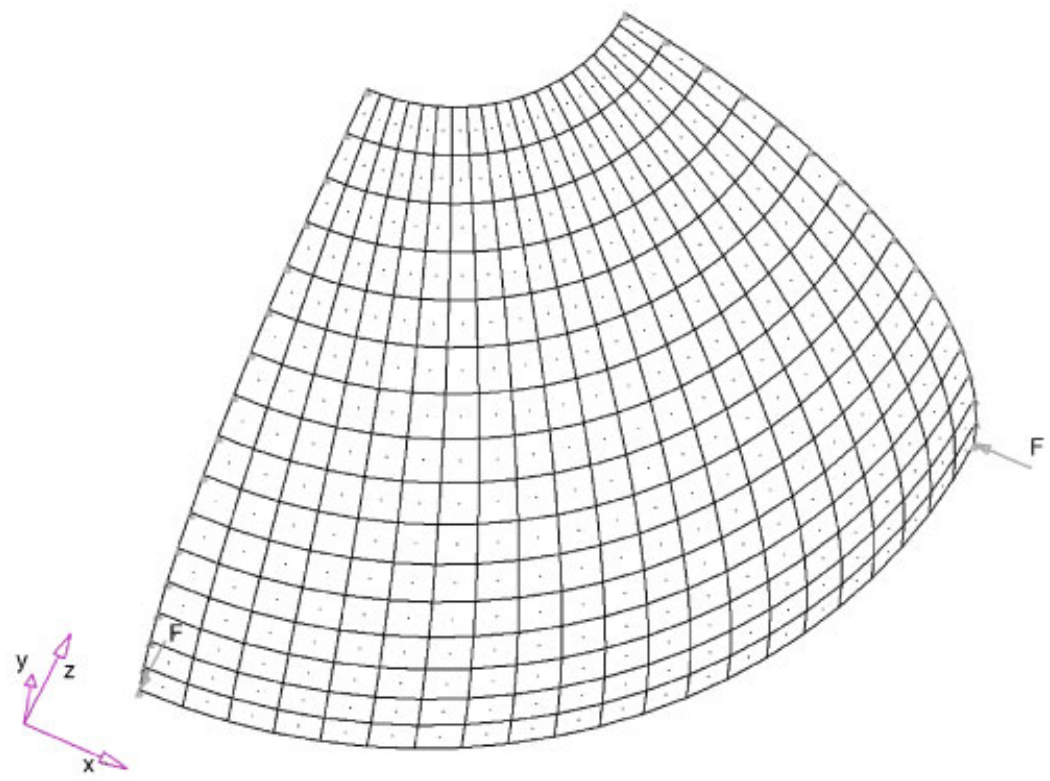

Figure 7. Finite element model for $18^{\circ}$ pinched hole hemisphere $(E=6.83 \mathrm{e} 7, v=0.3, t=0.04)$.

Table VIII. The results of $18^{\circ}$ pinched hole hemisphere.

\begin{tabular}{lccc}
\hline & $T x$ & $T y$ & $T z$ \\
\hline In-house (mixed) & $\mathbf{9 . 5 6 9 e - 2}$ & $1.011 \mathrm{e}-2$ & 0.0 \\
MSC/NASTRAN & $\mathbf{9 . 3 5 5 e - 2}$ & $5.336 \mathrm{e}-2$ & 0.0 \\
ANSYS & $\mathbf{9 . 3 0 0 e - 2}$ & $5.540 \mathrm{e}-2$ & 0.0 \\
ABAQUS & $\mathbf{9 . 2 9 4 e - 2}$ & $4.901 \mathrm{e}-2$ & 0.0 \\
\hline
\end{tabular}

Table IX. The results of open section beam.

\begin{tabular}{lccc}
\hline & $T x$ & $T y$ & $T z$ \\
\hline In-house (mixed) & $-2.440 \mathrm{e}-3$ & $\mathbf{- 1 . 1 5 3 e - 3}$ & $-1.418 \mathrm{e}-4$ \\
MSC/NASTRAN & $-2.433 \mathrm{e}-3$ & $\mathbf{- 1 . 1 5 4 e - 3}$ & $-1.417 \mathrm{e}-4$ \\
ANSYS & $-2.432 \mathrm{e}-3$ & $\mathbf{- 1 . 1 5 4 e - 3}$ & $-1.417 \mathrm{e}-4$ \\
ABAQUS & $-2.53 \mathrm{e}-3$ & $\mathbf{- 1 . 1 5 4 e - 3}$ & $-1.417 \mathrm{e}-4$ \\
\hline
\end{tabular}

three modal results from modal analysis are compared between shell model and solid model in Table X. For the $0.01 \mathrm{~mm}$ thickness, the results of shell model and solid model are similar, but they are different for the $1 \mathrm{~mm}$ thickness. Especially, the third natural frequency and modeshape of the $1 \mathrm{~mm}$ thickness models are different between shell model and solid model.

Table XI shows the results of in-house program-mixed shell element for seven-parameter formulation. First mode is bending and next two modes are torsion modes. There is no difference 


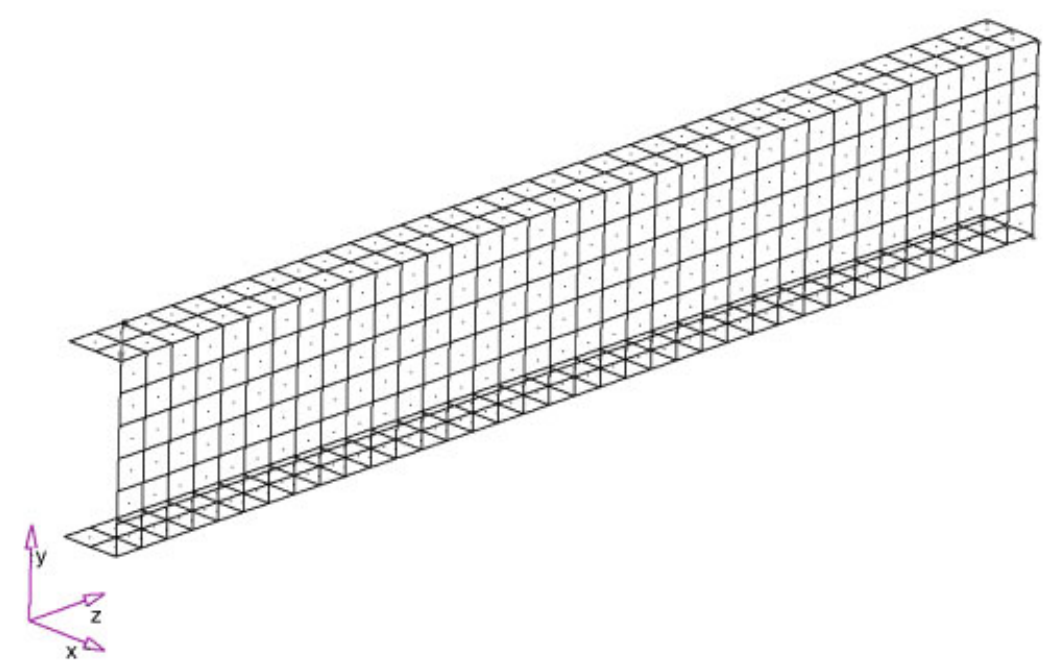

Figure 8. Finite element model for open section beam $(E=1.0 \mathrm{e} 7, v=0.333, t=0.05)$.
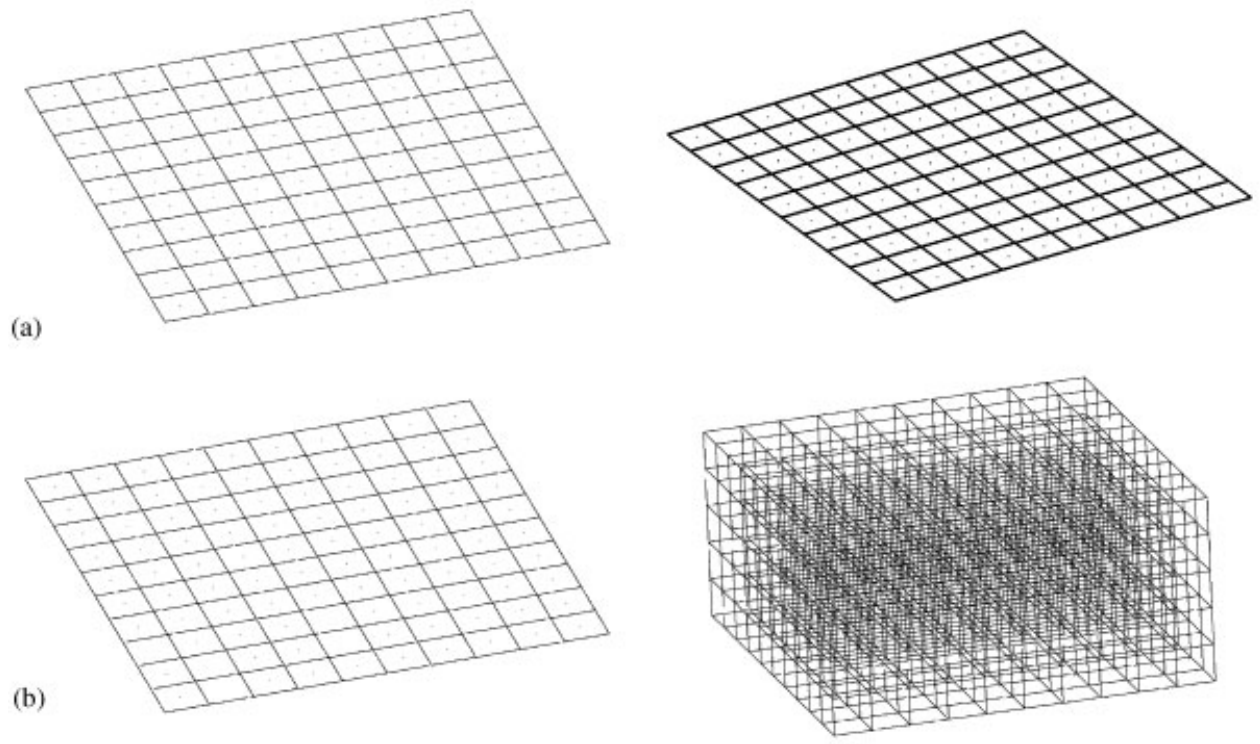

Figure 9. Finite element models of plate with 0.01 and $1 \mathrm{~mm}$ thickness $(E=2.0 \mathrm{e} 5, v=0.3)$ : (a) models of thickness $0.01 \mathrm{~mm}$ (shell (left) and solid (right) elements); and (b) models of thickness $1.0 \mathrm{~mm}$ (shell (left) and solid (right) elements).

between the results of shell model and solid model. The third natural frequency and mode shape of $1 \mathrm{~mm}$ thickness shell model are close to those of solid model. The results of inhouse program are also close to those of MSC/NASTRAN. Even though the eigenvalue of in-house program is a little less than that of solid element model and MSC/NASTRAN, they 
Table X. First three natural frequencies and modeshapes by MSC/NASTRAN.

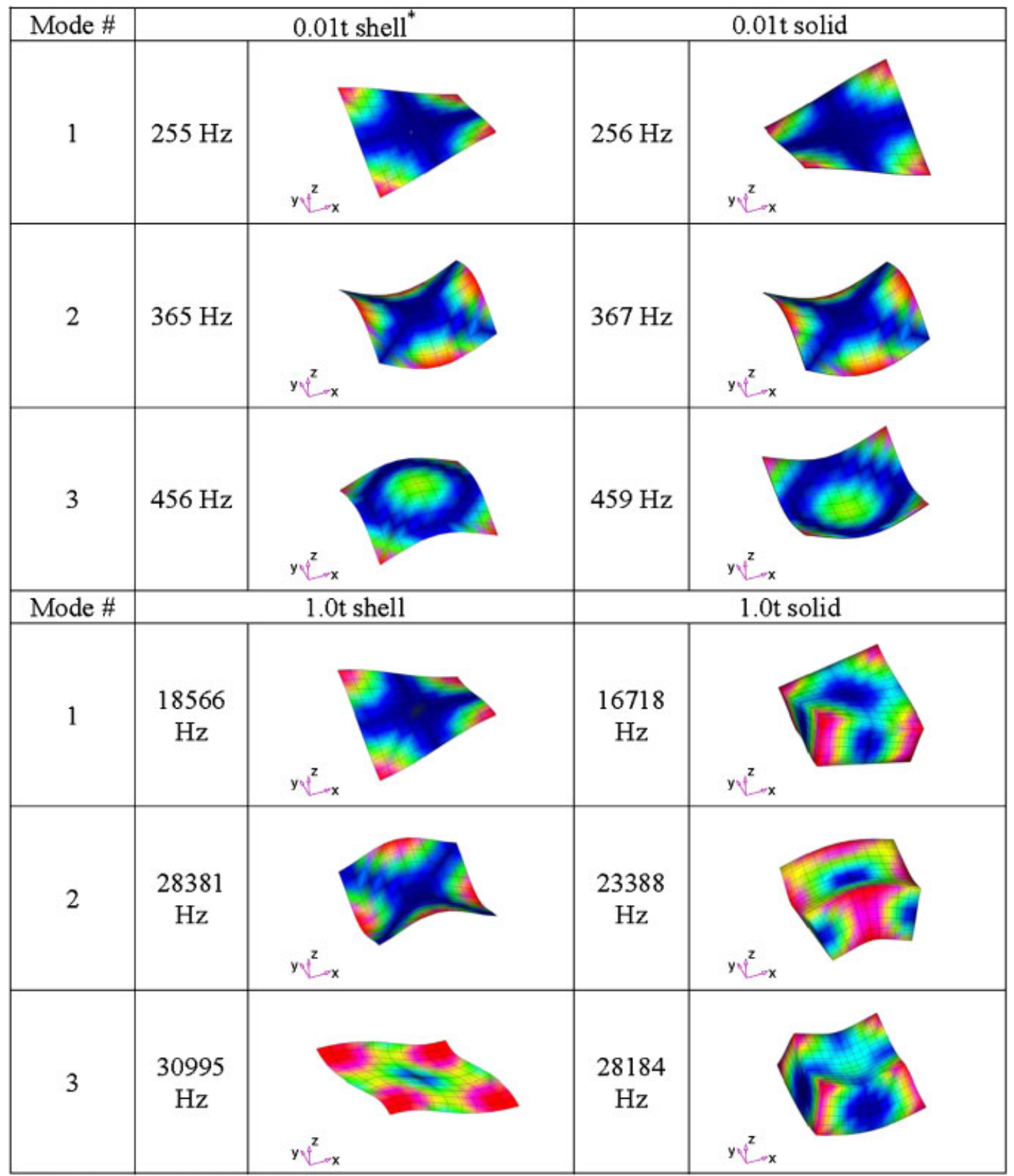

* $0.01 \mathrm{t}$ stands for $0.01 \mathrm{~mm}$ thickness 
Table XI. First three natural frequencies and modeshapes by in-house program.

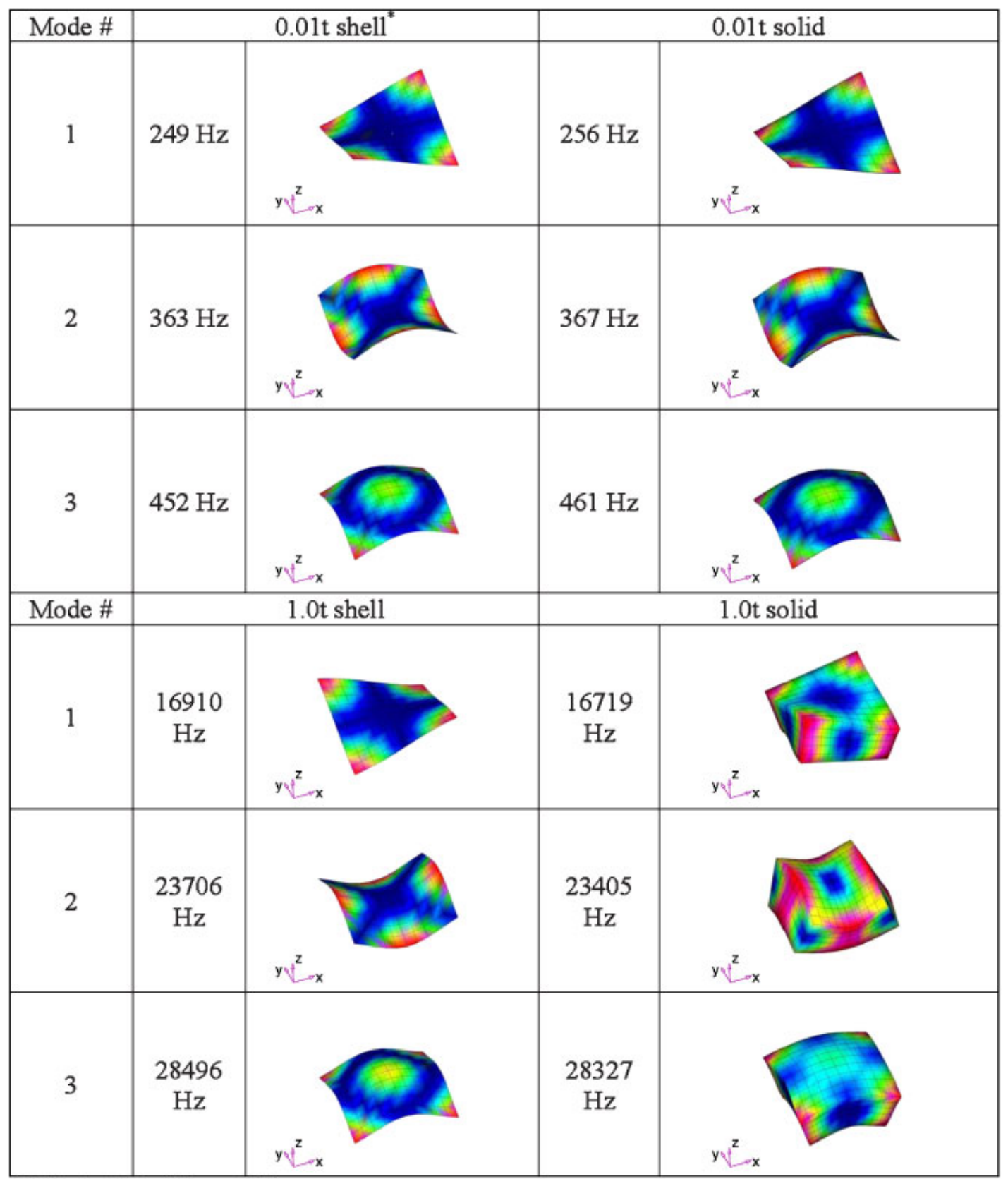

* $0.01 \mathrm{t}$ stands for $0.01 \mathrm{~mm}$ thickness 


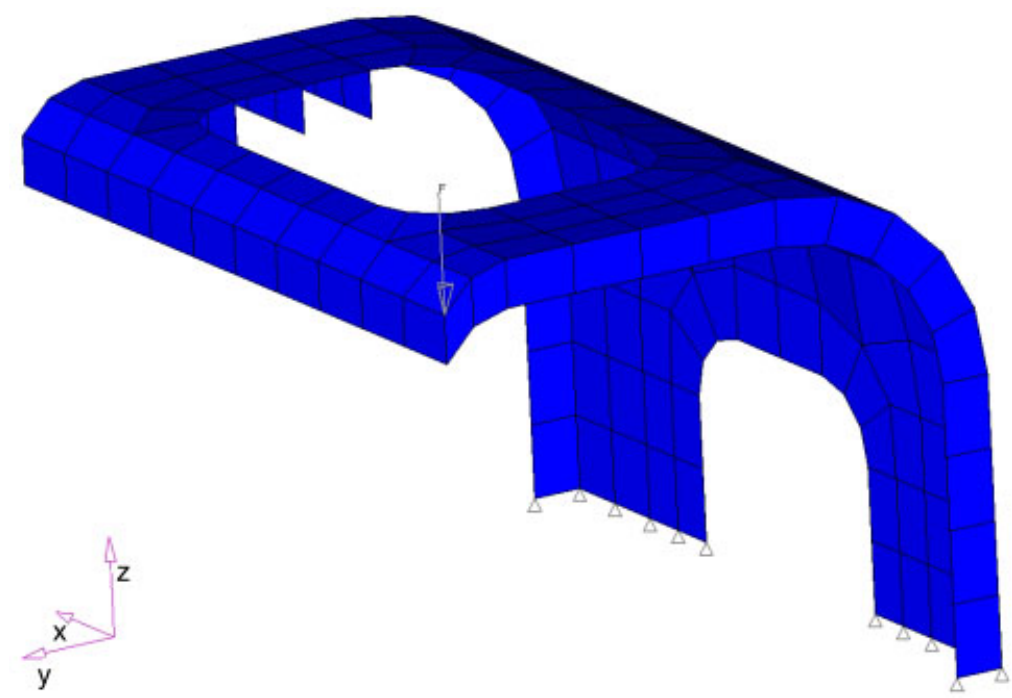

Figure 10. Bracket finite element model $(E=3.0 \mathrm{e} 7, v=0.3, t=0.05)$.

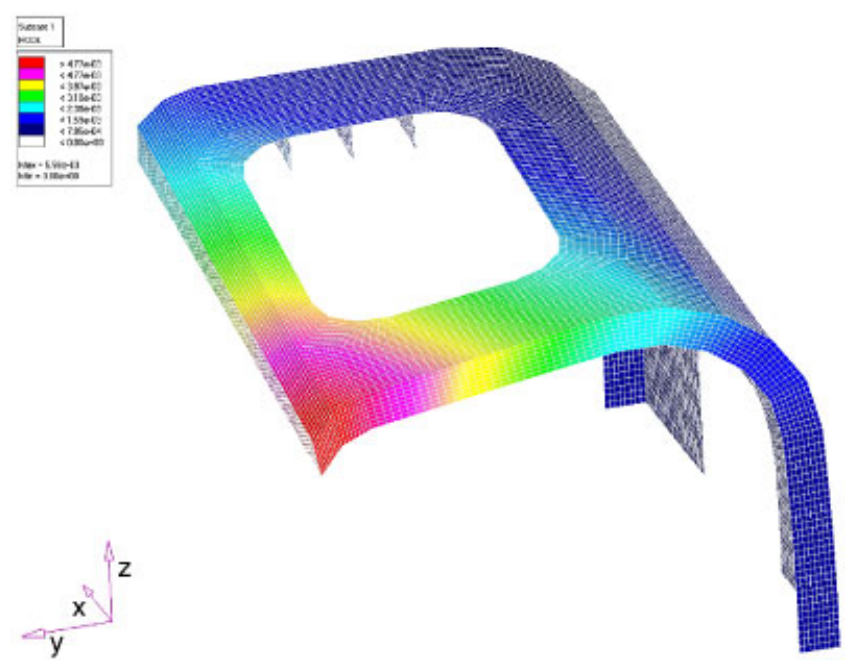

Figure 11. Deformed contour result of bracket model.

are acceptable results since the difference is not severe. Therefore, it is obvious that inhouse program developed in this paper can be used without distinction of the thin and thick shells. (The solid element is also implemented with selective reduced integration method in this paper. The results of solid model in Table XI come from the developed solid element). 


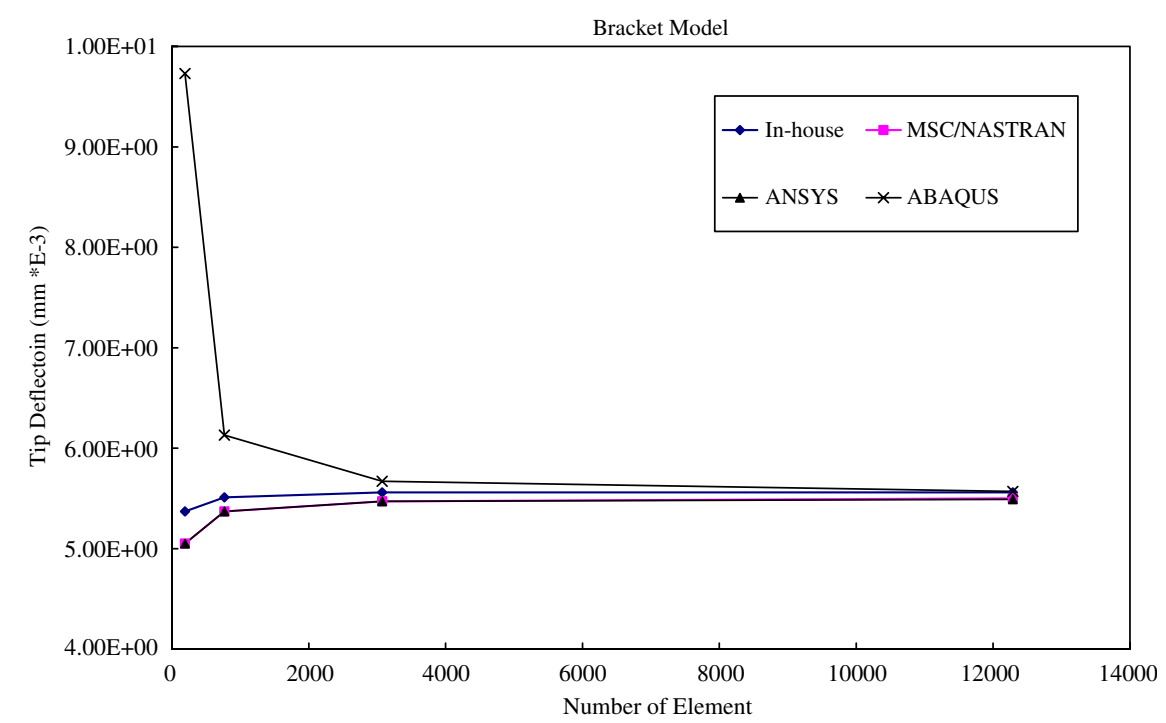

Figure 12. Convergence of tip deflection according to the number of element.

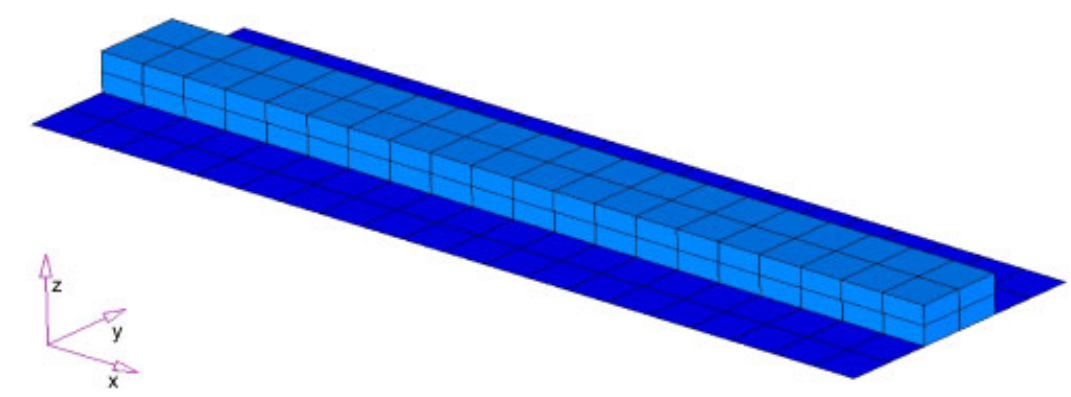

Figure 13. Finite element model of solid and shell combination model $(E=2.0 \mathrm{e} 8, v=0.3, t=0.1)$.

Bracket convergence test

In order to consider practical example, bracket model is considered as shown in Figure 10. End of bracket is constrained and one of the edge tips is applied by unit force. This example can be tested for bending and twisting performance of curved shell structure. Four different models that have different number of elements are tested. All models are converged as the number of elements increases as shown in Figure 12. ABAQUS has the worst performance for this example. Figure 11 presents the in-house contour results of 12288 elements model. 
Table XII. Natural frequencies of 200 elements model.

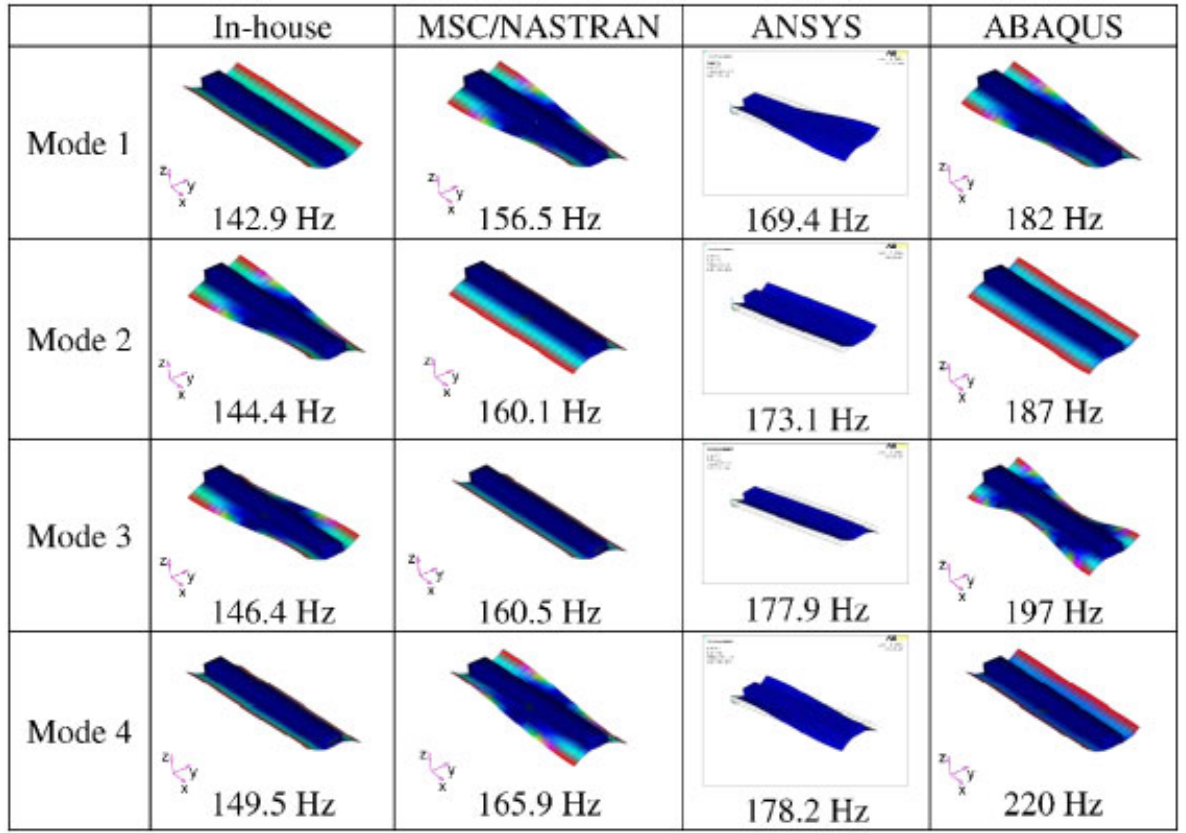

Table XIII. Natural frequencies of 1120 elements model.

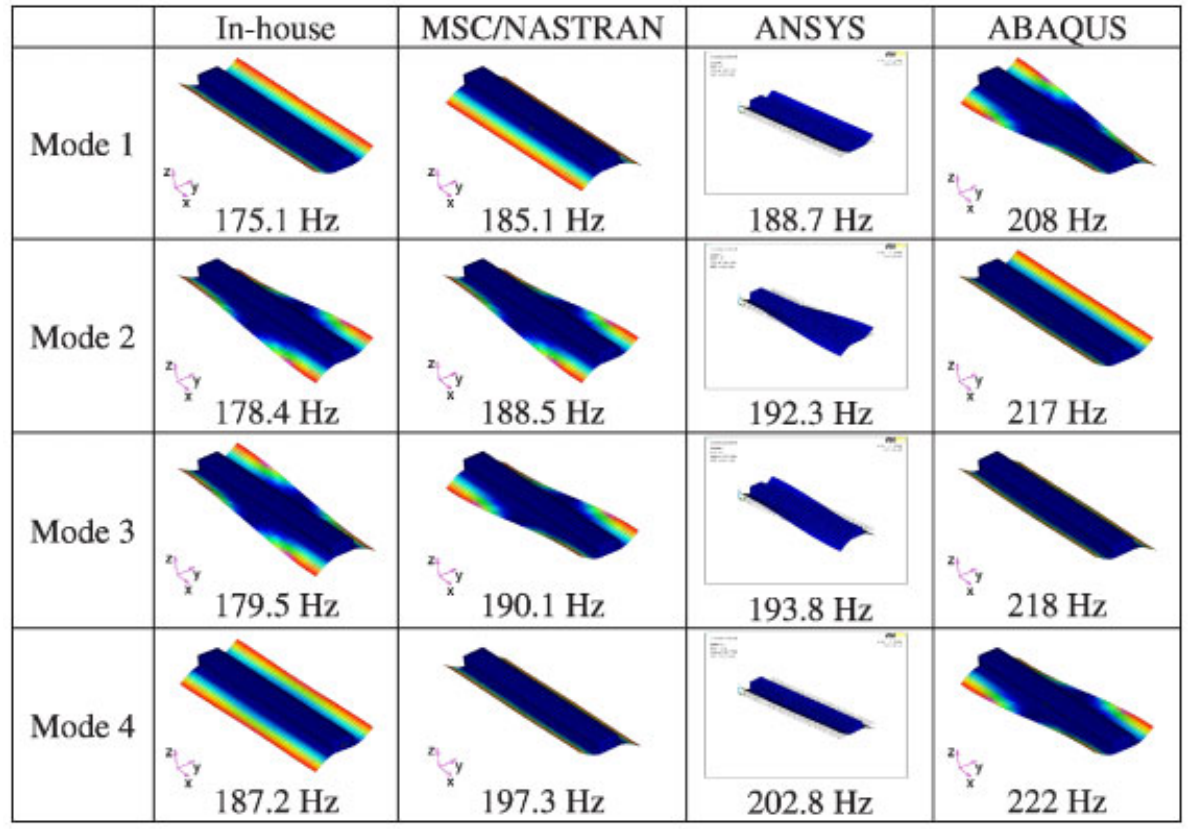


Table XIV. Natural frequencies of 7040 elements model.

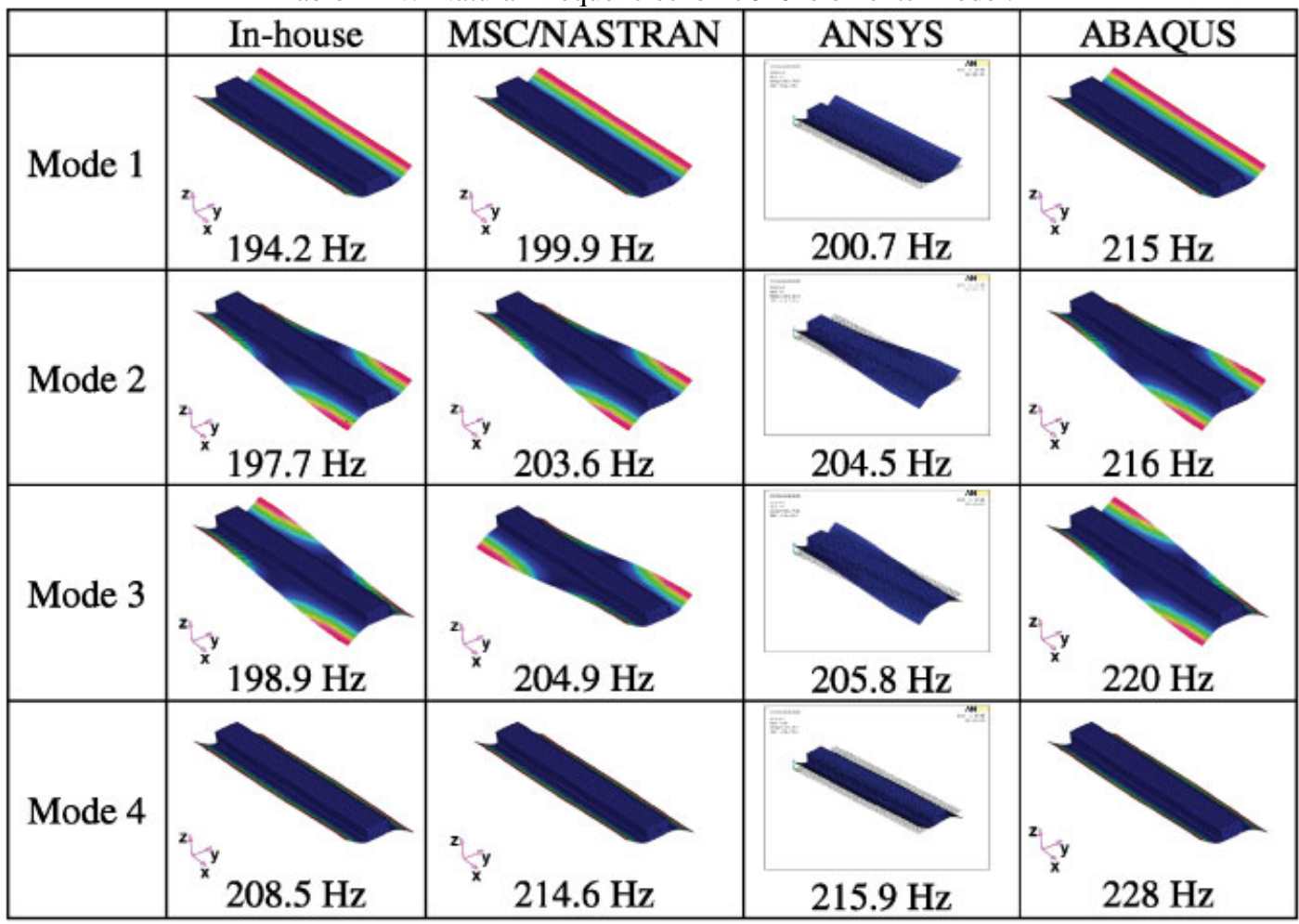

Solid and shell combination model

The model is shown in Figure 13. All modeshapes and its frequency are shown in Tables XII-XIV. In Table XII, there is difference for the first mode. Considering the geometry of the model, the first mode should be bending-not torsion. Therefore, in-house program provides better performance for modal analysis than other commercial software. For the results of ABAQUS, it is the worst among the results. Even though the number of elements is around 1000, ABAQUS provides strange first modeshape. Figures 14-17 show the convergence trend at each mode.

\section{CONCLUSION}

A new mixed shell element for seven-parameter formulation is developed in this paper. The developed element using seven-parameter formulation can be used without distinction of thin shell and thick shell. Even though the developed shell element cannot pass the patch test, the convergence can be obtained through various examples. Moreover, the robust modeshape can be obtained by in-house program for cantilever model of shell and solid combinations. Other examples present a reliable solution for the developed mixed shell element for seven-parameter formulation. 


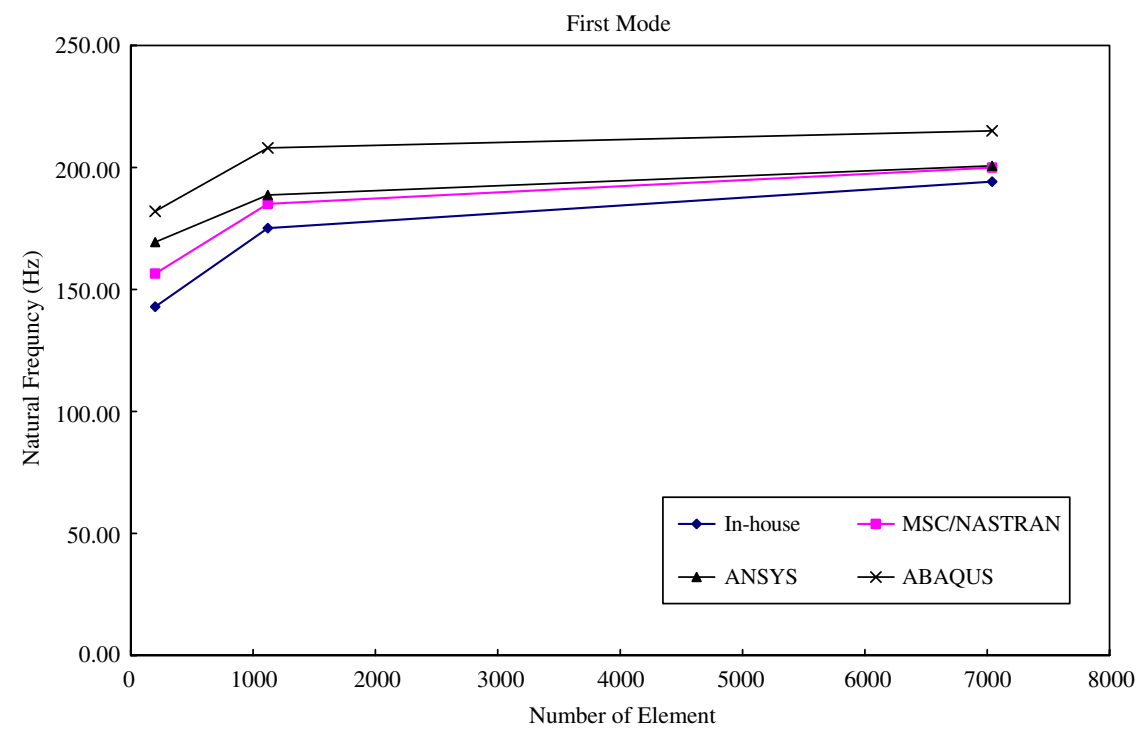

Figure 14. Convergence of first eigenvalues according to the number of element.

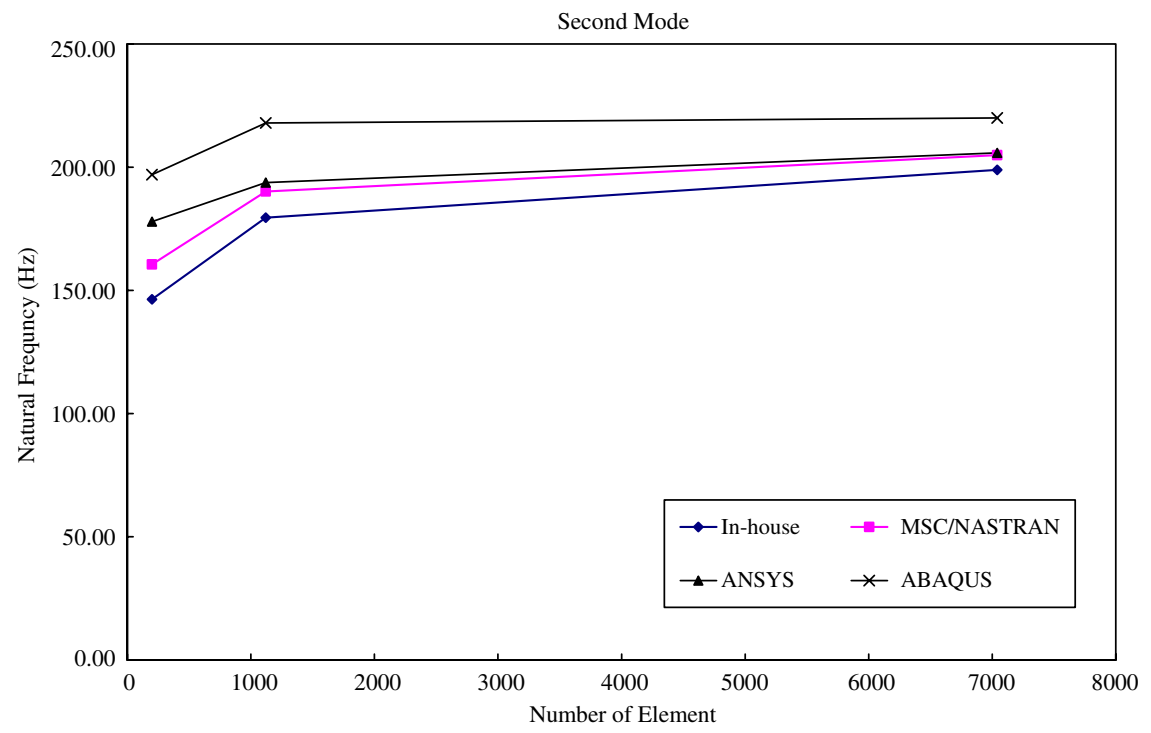

Figure 15. Convergence of second eigenvalues according to the number of element. 


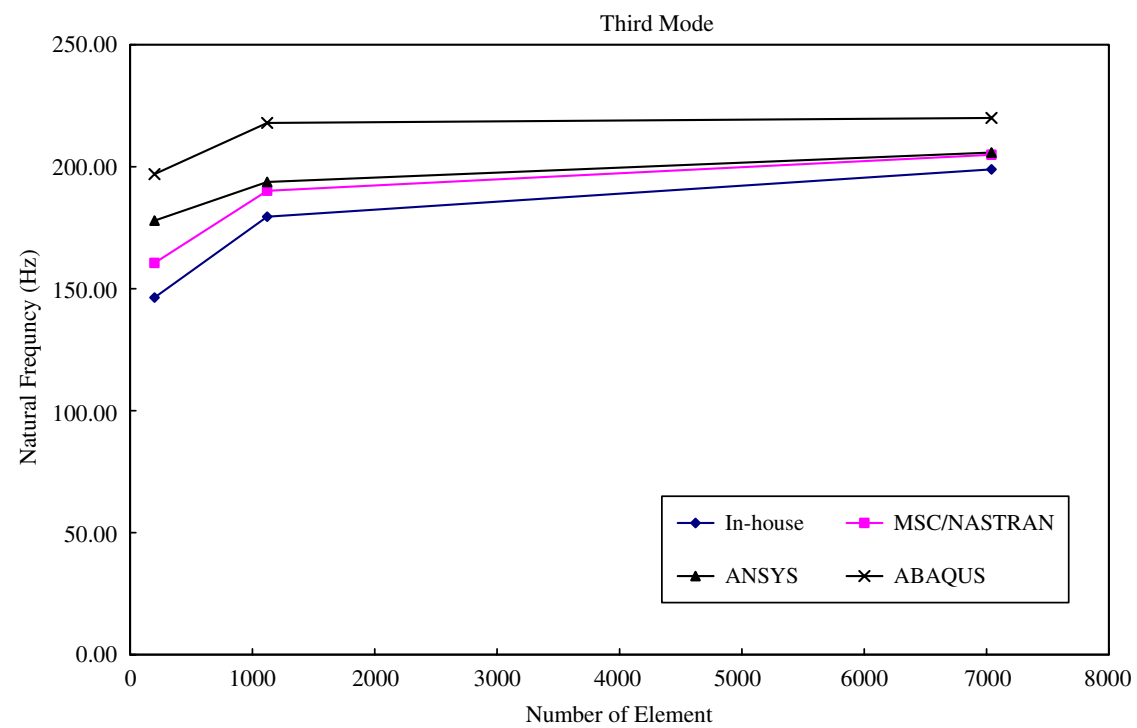

Figure 16. Convergence of third eigenvalues according to the number of element.

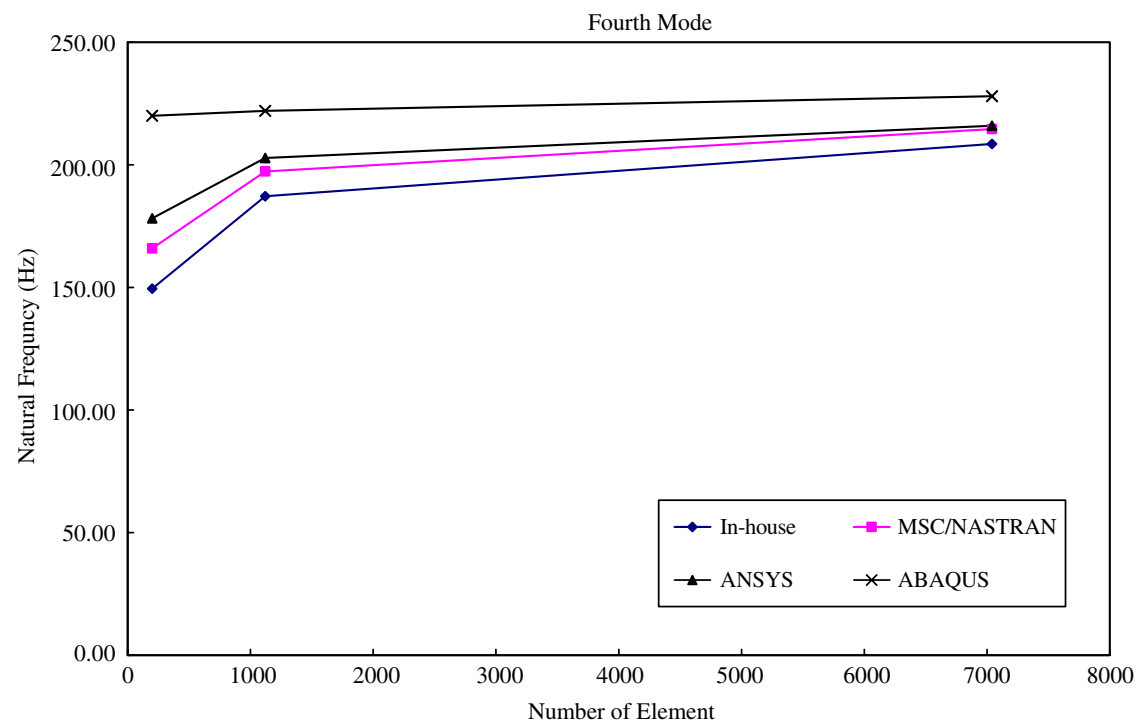

Figure 17. Convergence of fourth eigenvalues according to the number of element. 


\section{APPENDIX A}

Equation (32):

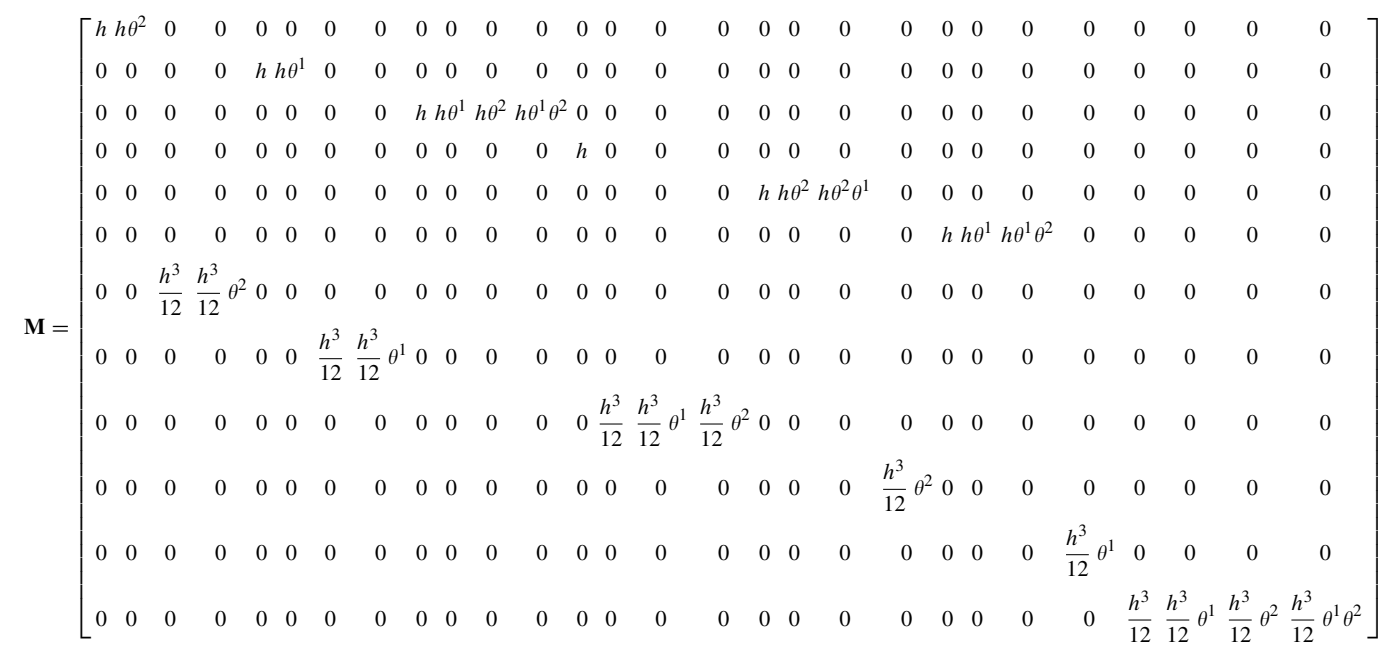

Equation (35):

The assumed stress can be also expressed as follows:

$$
\boldsymbol{\sigma}=\mathbf{S}=\mathbf{S}_{\mathbf{n}}+\theta^{3} \mathbf{S}_{\mathbf{m}}
$$

where

$$
\mathbf{S}_{\mathbf{n}}=\left(\begin{array}{l}
c_{1}+c_{2} \theta^{2} \\
c_{5}+c_{6} \theta^{1} \\
c_{9}+c_{10} \theta^{1}+c_{11} \theta^{2}+c_{12} \theta^{1} \theta^{2} \\
c_{13} \\
c_{17}+c_{18} \theta^{2}+c_{19} \theta^{2} \theta^{1} \\
c_{21}+c_{22} \theta^{1}+c_{23} \theta^{1} \theta^{2}
\end{array}\right), \quad \mathbf{S}_{\mathbf{m}}=\left(\begin{array}{l}
c_{3}+c_{4} \theta^{2} \\
c_{7}+c_{8} \theta^{1} \\
c_{25}+c_{26} \theta^{1}+c_{27} \theta^{2}+c_{28} \theta^{1} \theta^{2} \\
c_{14}+c_{15} \theta^{1}+c_{16} \theta^{2} \\
c_{20} \theta^{2} \\
c_{24} \theta^{1}
\end{array}\right)
$$

Equation (A2) is another expression of Equation (32). Using Equations (A2) and (A3), the following form for assumed stress can be defined:

$$
\begin{aligned}
\int \delta \boldsymbol{\sigma}^{\mathrm{T}} \mathbf{D}^{-1} \boldsymbol{\sigma} \mathrm{d} \Omega & =\int \delta\left(\mathbf{S}_{\mathbf{n}}+\theta^{3} \mathbf{S}_{\mathbf{m}}\right)^{\mathrm{T}} \mathbf{D}^{-1}\left(\mathbf{S}_{\mathbf{n}}+\theta^{3} \mathbf{S}_{\mathbf{m}}\right) \mathrm{d} \Omega \\
& =\int \delta \mathbf{S}_{\mathbf{n}}^{\mathrm{T}} h \mathbf{D}_{n}^{-1} \mathbf{S}_{\mathbf{n}}+\mathbf{S}_{\mathbf{m}}^{\mathrm{T}} \frac{h^{3}}{12} \mathbf{D}_{m}^{-1} \mathbf{S}_{\mathbf{m}} \mathrm{d} A \\
& =\delta \mathbf{c}^{\mathrm{T}} \int \mathbf{H}^{\mathrm{T}} \overline{\mathbf{D}}^{-1} \mathbf{H} \mathrm{d} A \mathbf{c}
\end{aligned}
$$


where

$$
\mathbf{H}=\left[\begin{array}{cccccccccccccccccccccccccccc}
1 & \theta^{2} & 0 & 0 & 0 & 0 & 0 & 0 & 0 & 0 & 0 & 0 & 0 & 0 & 0 & 0 & 0 & 0 & 0 & 0 & 0 & 0 & 0 & 0 & 0 & 0 & 0 & 0 \\
0 & 0 & 0 & 0 & 1 & \theta^{1} & 0 & 0 & 0 & 0 & 0 & 0 & 0 & 0 & 0 & 0 & 0 & 0 & 0 & 0 & 0 & 0 & 0 & 0 & 0 & 0 & 0 & 0 \\
0 & 0 & 0 & 0 & 0 & 0 & 0 & 0 & 1 & \theta^{1} & \theta^{2} & \theta^{1} \theta^{2} & 0 & 0 & 0 & 0 & 0 & 0 & 0 & 0 & 0 & 0 & 0 & 0 & 0 & 0 & 0 & 0 \\
0 & 0 & 0 & 0 & 0 & 0 & 0 & 0 & 0 & 0 & 0 & 0 & 1 & 0 & 0 & 0 & 0 & 0 & 0 & 0 & 0 & 0 & 0 & 0 & 0 & 0 & 0 & 0 \\
0 & 0 & 0 & 0 & 0 & 0 & 0 & 0 & 0 & 0 & 0 & 0 & 0 & 0 & 0 & 0 & 1 & \theta^{2} & \theta^{1} \theta^{2} & 0 & 0 & 0 & 0 & 0 & 0 & 0 & 0 & 0 \\
0 & 0 & 0 & 0 & 0 & 0 & 0 & 0 & 0 & 0 & 0 & 0 & 0 & 0 & 0 & 0 & 0 & 0 & 0 & 0 & 1 & \theta^{1} & \theta^{1} \theta^{2} & 0 & 0 & 0 & 0 & 0 \\
0 & 0 & 1 & \theta^{2} & 0 & 0 & 0 & 0 & 0 & 0 & 0 & 0 & 0 & 0 & 0 & 0 & 0 & 0 & 0 & 0 & 0 & 0 & 0 & 0 & 0 & 0 & 0 & 0 \\
0 & 0 & 0 & 0 & 0 & 0 & 1 & \theta^{1} & 0 & 0 & 0 & 0 & 0 & 0 & 0 & 0 & 0 & 0 & 0 & 0 & 0 & 0 & 0 & 0 & 0 & 0 & 0 & 0 \\
0 & 0 & 0 & 0 & 0 & 0 & 0 & 0 & 0 & 0 & 0 & 0 & 0 & 1 & \theta^{1} & \theta^{2} & 0 & 0 & 0 & 0 & 0 & 0 & 0 & 0 & 0 & 0 & 0 & 0 \\
0 & 0 & 0 & 0 & 0 & 0 & 0 & 0 & 0 & 0 & 0 & 0 & 0 & 0 & 0 & 0 & 0 & 0 & 0 & \theta^{2} & 0 & 0 & 0 & 0 & 0 & 0 & 0 & 0 \\
0 & 0 & 0 & 0 & 0 & 0 & 0 & 0 & 0 & 0 & 0 & 0 & 0 & 0 & 0 & 0 & 0 & 0 & 0 & 0 & 0 & 0 & 0 & \theta^{1} & 0 & 0 & 0 & 0 \\
0 & 0 & 0 & 0 & 0 & 0 & 0 & 0 & 0 & 0 & 0 & 0 & 0 & 0 & 0 & 0 & 0 & 0 & 0 & 0 & 0 & 0 & 0 & 0 & 1 & \theta^{1} & \theta^{2} & \theta^{1} \\
\theta^{2}
\end{array}\right]
$$

(A5)

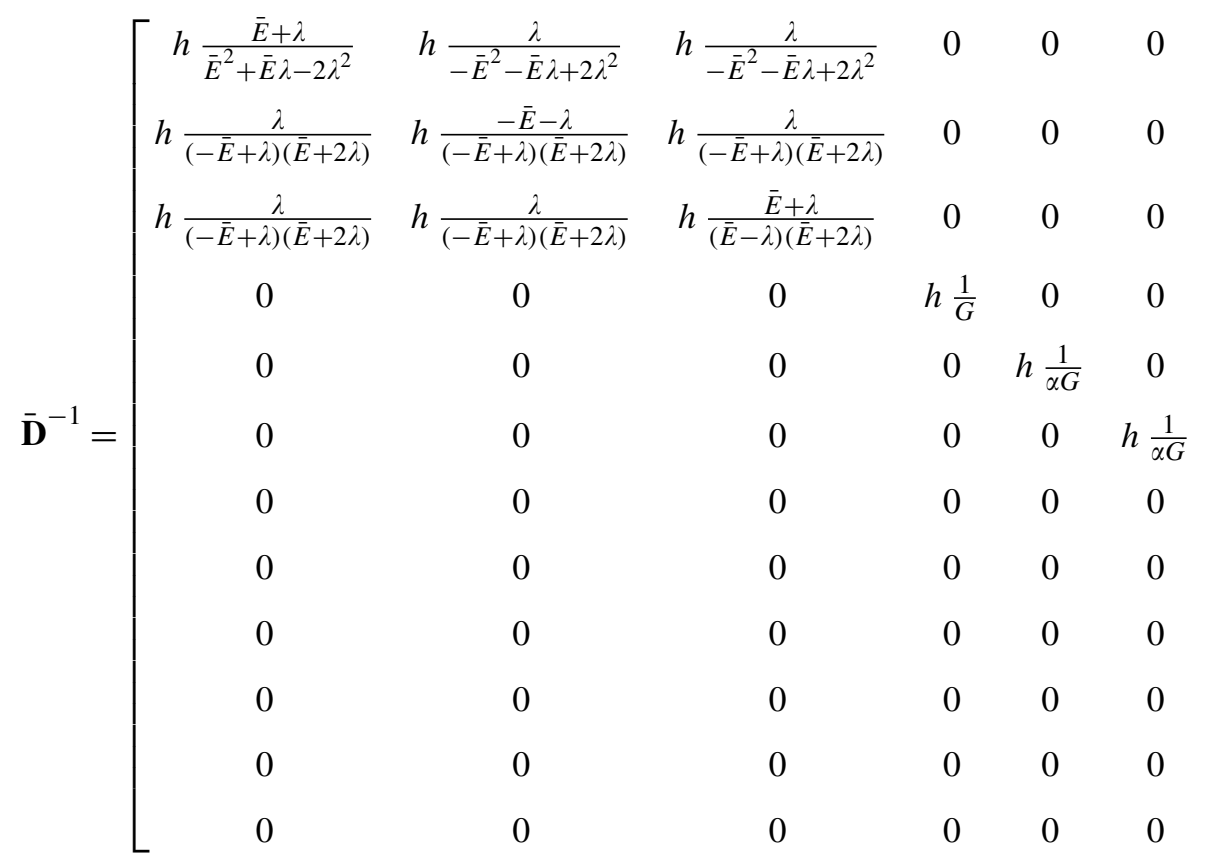




$\left.\begin{array}{cccccc}0 & 0 & 0 & 0 & 0 & 0 \\ 0 & 0 & 0 & 0 & 0 & 0 \\ 0 & 0 & 0 & 0 & 0 & 0 \\ 0 & 0 & 0 & 0 & 0 & 0 \\ 0 & 0 & 0 & 0 & 0 & 0 \\ 0 & 0 & 0 & 0 & 0 & 0 \\ \frac{h^{3}}{12} \frac{\bar{E}+\lambda}{\bar{E}^{2}+\bar{E} \lambda-2 \lambda^{2}} & \frac{h^{3}}{12} \frac{\lambda}{-\bar{E}^{2}-\bar{E} \lambda+2 \lambda^{2}} & 0 & 0 & 0 & \frac{h^{3}}{12} \frac{\lambda}{-\bar{E}^{2}-\bar{E} \lambda+2 \lambda^{2}} \\ \frac{h^{3}}{12} \frac{\lambda}{(-\bar{E}+\lambda)(\bar{E}+2 \lambda)} & \frac{h^{3}}{12} \frac{-\bar{E}-\lambda}{(-\bar{E}+\lambda)(\bar{E}+2 \lambda)} & 0 & 0 & 0 & \frac{h^{3}}{12} \frac{\lambda}{(-\bar{E}+\lambda)(\bar{E}+2 \lambda)} \\ 0 & 0 & \frac{h^{3}}{12} \frac{1}{G} & 0 & 0 & 0 \\ \frac{\lambda}{0} & 0 & 0 & \frac{h^{3}}{12} \frac{1}{\beta G} & 0 & 0 \\ \frac{h^{3}}{12} \frac{\lambda}{(-\bar{E}+\lambda)(\bar{E}+2 \lambda)} & \frac{h^{3}}{12} \frac{\lambda}{(-\bar{E}+\lambda)(\bar{E}+2 \lambda)} & 0 & 0 & 0 & \frac{h^{3}}{12} \frac{\bar{E}+\lambda}{(\bar{E}-\lambda)(\bar{E}+2 \lambda)}\end{array}\right]$

(A6)

\section{ACKNOWLEDGEMENTS}

We would like to express special thanks to ARC (Automotive Research Center) for supporting this research.

\section{REFERENCES}

1. Ahmad S, Irons BM, Zienkiewicz OC. Analysis of thick and shell structures by curved finite element. International Journal for Numerical Methods in Engineering 1990; 2:419-459.

2. Argyris JH, Scharpf DW. The SHEBA family of shell elements for the matrix displacement method. Journal of the Royal Aeronautical Society 1968; 72:873-883.

3. Argyris JH, Papadrakakis M, Apostolopoulou C, Koutsourelakis S. The TRIC shell element: theoretical and numerical investigation. Computer Methods in Applied Mechanics and Engineering 2000; 182:217-245.

4. Hughes TJR, Cohen M, Maroun M. Reduced and selective integration techniques in finite element analysis of plates. Nuclear Engineering and Design 1978; 46:203-222.

5. Belytschko T, Tsay C. A stabilization procedure for the quadrilateral plate element with one-point quadrature. International Journal for Numerical Methods in Engineering 1983; 19:405-419.

6. Simo JC, Rifai MS. A class of mixed assumed strain method of incompatible modes. International Journal for Numerical Methods in Engineering 1990; 29:1595-1638.

7. Simo JC, Armero F, Taylor RL. Improved versions of assumed enhanced strain tri-linear elements or 3D finite deformation problems. Computer Methods in Applied Mechanics and Engineering 1993; 110:359-386.

8. MacNeal RH. A simple quadrilateral shell element. Computers and Structures 1978; 8:175-183.

9. Bathe KJ, Iosilevich A, Chapelle D. An evaluation of the MITC shell elements. Computers and Structures 2000; 75(1):1-30. 
10. Chapelle D, Oliveira DL, Bucalem ML. MITC elements for a classical shell model. Computers and Structures 2003; 81(8-11):523-533.

11. Clough RW. The finite element method in plane stress analysis. Proceedings of 2nd Conference on Electronic Computation, 1960; 345-378.

12. Pian THH. Derivation of element stiffness matrices by assumed stress distributions. American Institute of Architects Architecture Journal 1964; 2:1333-1376.

13. Pian THH, Sumihara K. Rational approach for assumed stress finite elements. International Journal for Numerical Methods in Engineering 1984; 20:1685-1695.

14. Sekiguchi M. A study of element distortion for membranes and plates. Ph.D. Dissertation, University of Tokyo, 2003.

15. Bischoff M, Ramm E. On the physical significance of higher order kinematic and static variables in a three-dimensional shell formulation. International Journal of Solids and Structures 2000; 37:6933-6960.

16. Büchter N, Ramm E, Roehl D. Three-dimensional extension of nonlinear shell formulation based on the enhanced assumed strain concept. International Journal for Numerical Methods in Engineering 1994; 37:2551-2568.

17. Reissner E. The effect of transverse shear deformation on the bending of elastic plates. Journal of Applied Mechanics Transaction (ASME) 1945; 12:A69-A77.

18. Mindlin RD. Influence of rotatory inertia and shear on flexural motions of isotropic elastic plates. Journal of Applied Mechanics 1951; 18:31-38.

19. Schlebusch R, Matheas J, Zastrau BW. A three-dimensional surface-related shell theory for the treatment of contact problems. Fifth World Congress on Computational Mechanics, Vienna, Austria, 7-12 July 2002.

20. Weissman SL, Taylor RL. Treatment of internal constraints by mixed finite element methods: unification of concept. International Journal for Numerical Methods in Engineering 1992; 33:131-141.

21. Wall WA, Gee M, Ramm E. The challenge of a three-dimensional shell formulation-the conditioning problem. Proceedings of 'IASS-IACM 2000', Fourth International Colloquium on Computation of Shell and Spatial Structures, Chania, Crete, Greece, 4-7 June 2000.

22. Bischoff M, Taylor RL. A three-dimensional shell element with an exact thin limit. European Conference on Computational Mechanics, Cracow, Poland, 2001.

23. Sansour C, Kollmann FG. Families of 4-node and 9-node finite elements for a finite deformation shell theory. An assessment of hybrid stress, hybrid strain and enhanced strain elements. Computational Mechanics 2000; 24:435-447.

24. Sansour C, Bocko J. On hybrid stress, hybrid strain and enhanced strain finite element formulations for a geometrically exact shell theory with drilling degrees of freedom. International Journal for Numerical Methods in Engineering 1998; 43:175-192.

25. Betsch P, Gruttmann F, Stein E. A 4-node finite shell element for the implementation of general hyperelastic 3D-elasticity at finite strains. Computer Methods in Applied Mechanics and Engineering 1996; 130:57-79.

26. MacNeal RH. A proposed standard set of problems to test finite element accuracy. Finite Element in Analysis and Design 1985; 1:3-20. 